\title{
Heterogeneidade altitudinal na Floresta Atlântica setentrional: um estudo de caso no sul da Bahia, Brasil
}

\author{
Diogo Souza Bezerra Rocha ${ }^{1,3,4}$ e André Márcio Araujo Amorim²,3
}

Recebido em 1/02/2011. Aceito em 7/02/2012

\begin{abstract}
RESUMO
(Heterogeneidade altitudinal na Floresta Atlântica setentrional: um estudo de caso no sul da Bahia, Brasil) A Floresta Atlântica apresenta padrões de heterogeneidade ainda pouco conhecidos, em especial aqueles associados à altitude, raramente investigados no eixo setentrional brasileiro. O objetivo do presente trabalho foi verificar a ocorrência de variações na composição florística, diversidade e estrutura da vegetação num remanescente de floresta Montana no sul da Bahia, através da análise de dois trechos de vegetação em diferentes altitudes. Foram amostrados no total 0,5 ha através de cinco plots separados entre si e alocados numa floresta úmida na RPPN Serra Bonita, Bahia, Brasil. Registraram-se 354 espécies de 68 famílias, sendo Myrtaceae, Fabaceae, Rubiaceae e Lauraceae as mais ricas. Os dois trechos apresentaram diferenças em densidade, área basal e estrutura, percebendo-se que com o aumento da altitude, ocorre uma diminuição no porte da floresta, refletindo maior densidade no sub-bosque, além de redução na riqueza específica. A Serra Bonita apresentou alta diversidade, similar a outras áreas na Bahia, porém maior que na Floresta Atlântica no sudeste do Brasil. Os trechos amostrados apresentaram bom estado de conservação, com abundância de lianas lenhosas, elevada área basal e baixa evidência de extração madeireira. As variações detectadas entre os trechos inventariados sugerem modificações estruturais abruptas, influenciadas possivelmente por precipitação, temperatura e barreiras locais determinadas por uma topografia acidentada, mas não por fatores edáficos.
\end{abstract}

Palavras-chave: diversidade arbórea, floresta Montana, perfil de diversidade, curvas de rarefação, similaridade

\begin{abstract}
(Altitudinal heterogeneity in northern Atlantic Forest: a case study in southeastern Bahia, Brazil) The Atlantic Forest has a heterogeneity that is still largely unappreciated, especially that associated with altitudinal factors; this has rarely been examined in the northern extension of this biome. The study investigated the occurrence of variation in floristic composition, diversity, and vegetation structure at two altitudes in a remnant area of forest in southern Bahia state. A total of 0.5 ha of vegetation was sampled in five separate plots established in a wet forest in the RPPN Serra Bonita, Bahia, Brazil. A total of 354 species belonging to 68 families were found, with Myrtaceae, Fabaceae, Rubiaceae and Lauraceae being the richest taxa. The two altitudinal sites showed significant differences in density, basal area and structure. There were notable changes in forest structure with increasing altitude, reflected in higher densities of the understory and a reduction in species richness. Serra Bonita had high species diversity that was similar to other areas in Bahia, but higher than found in the Atlantic Forests of southeastern Brazil. The forest sections sampled were well preserved, as indicated by the abundance of woody vines, elevated basal area, and lack of evidence of significant logging activities. The variation detected between the areas suggests the existence of abrupt structural modifications influenced not by edaphic factors but by rainfall differences, temperature variations, and by local barriers determined by the steep topography.
\end{abstract}

Key words: tree diversity, montane forest, diversity profiles, rarefaction curves, similarity

1 Universidade Estadual de Feira de Santana, Programa de Pós-Graduação em Botânica, Departamento de Ciências Biológicas, Feira de Santana, BA, Brasil

2 Universidade Estadual de Santa Cruz, Departamento de Ciências Biológicas, Ilhéus, BA, Brasil

3 Herbário CEPEC/CEPLAC, Ilhéus, BA, Brasil

4 Autor para correspondência: diogosbr@gmail.com 


\section{Introdução}

A Floresta Atlântica possui composição florística e fitofisionomia variável ao longo de toda a sua extensão, apresentando condições climáticas, topografia e geomorfologia diversificada (Oliveira-Filho \& Fontes 2000; Oliveira-Filho et al. 2006). Este domínio fitogeográfico é intensamente explorado e degradado a mais de 500 anos (Morellato \& Haddad 2000), mas a preocupação com a conservação de sua biodiversidade é relativamente recente. Por este motivo, a floresta que inicialmente cobria cerca de $1.300 .000 \mathrm{~km}^{2}$, hoje está reduzida a menos de $8 \%$ de sua cobertura original (Morellato \& Haddad 2000; Oliveira-Filho \& Fontes 2000) em decorrência de uma intensa ação antrópica. Esta floresta conhecida pelos altos níveis de endemismos e diversidade em espécies vegetais e animais, é considerada um dos 34 hotspots de biodiversidade mundial (Mittermeier et al. 2004).

No nordeste brasileiro, a Floresta Atlântica encontra-se ainda mais fragmentada, estimando-se na Bahia, por exemplo, menos de $4 \%$ de sua cobertura original (Thomas et al. 1998; Cassano et al. 2008). Grande parte das florestas úmidas neste estado foi modificada em áreas de produção de cacau (Theobroma cacau L.) desde o início do século XIX, através do sistema agroflorestal cabruca (Sambuichi 2002). O declínio na produção, a partir do final do século XX foi causado por infestações consecutivas de fungos sistêmicos, especialmente Moniliophthora perniciosa (Stahel) Aime \& Phillips-Mora. Aliado a isso, os baixos preços no mercado externo determinaram o abandono de diversas áreas resultando em uma redução na expansão da lavoura, mas também ocasionando a supressão total de cabrucas para implantação de pecuária extensiva (Cassano et al. 2008).

Os remanescentes de floresta ainda existentes, excetuando-se as áreas protegidas como Parques Nacionais e Reservas Biológicas, concentram-se nos topos de montanhas e/ou em encostas mais íngremes (Thomas et al. 1998; 2008; Amorim et al.2009), onde a atividade agropecuária é difícil ou inviável, seja pelo acesso ou pelos solos geralmente com baixa fertilidade (Moreno et al. 2003). Dessa forma, áreas de floresta situadas em altitudes, dentro da região produtora de cacau permaneceram mais protegidas. No entanto, as dificuldades que retardaram a degradação destas áreas também fizeram com que fossem pouco investigadas, resultando em um conhecimento biológico incipiente. Martinelli (2007) com o intuito de inserir o estudo da biodiversidade de montanhas em pesquisas científicas indica várias formações como áreas de extrema importância biológica e com necessidades prementes de inventários. Entre elas, é citada a Serra Bonita (= Serra da Panelinha) como um dos remanescentes prioritários em Floresta Atlântica no estado da Bahia.

A maior parte dos trabalhos sobre florística e estrutura de comunidades em florestas úmidas no sul da Bahia é concentrada em formações Submontanas (Mori et al. 1983; Martini et al. 2007; Amorim et al. 2008; Thomas et al. 2008).
Desta forma, as florestas Montanas apresentam escassez de informações até mesmo na documentação das espécies para fins taxonômicos e biogeográficos. Nos últimos anos foram realizados estudos com a intenção de se avançar no conhecimento florístico (e.g. Amorim et al. 2005; 2009; Matos 2009; Matos et al. 2010a; Mota et al. 2009) e de estrutura (Thomas et al. 2009; Mortara 2011) nestes ambientes. Resultados recentes revelaram a existência de espécies vasculares ainda não descritas (Amorim \& Leme 2009; Matos et al. 2009; 2010b), novos registros de endemismos e ocorrência de espécies ainda não conhecidas no nordeste do Brasil (Labiak \& Prado 2007; Amorim et al. 2009; Mota et al. 2009; Matos et al. 2010a) e indícios da existência de uma acentuada heterogeneidade vegetacional(Thomas et al. 2009).

Face ao exposto, objetivou-se nesse estudo investigar variações na composição florística, diversidade e estrutura da vegetação num fragmento de floresta Montana na RPPN Serra Bonita, através da análise de dois trechos de vegetação em diferentes altitudes, partindo da hipótese de que essa floresta apresenta uma modificação estrutural e fisionômica associada a fatores ligados à altitude (e.g. composição edáfica).

\section{Material e métodos}

\section{Área de estudo}

A Serra Bonita é um complexo de montanhas situada entre os municípios de Camacan e Pau-Brasil em coordenadas geográficas aproximadas entre $15^{\circ} 22^{\prime} 50^{\prime \prime} \mathrm{S}$ a $15^{\circ} 24^{\prime}$ $16^{\prime \prime} \mathrm{S}$ e $39^{\circ} 33^{\prime} 12^{\prime \prime} \mathrm{O}$ a $39^{\circ} 34^{\prime} 40^{\prime \prime} \mathrm{O}$, com ca. 7.500 ha de área total e altitudes que variam entre 300 a $1.080 \mathrm{~m}$ s.n.m. (Amorim et al. 2009), onde existe, na área central mais alta, a Reserva Particular do Patrimônio Natural - RPPN Serra Bonita, Camacan, Bahia (Fig. 1). Na RPPN e região contígua, o clima é considerado do tipo Af (Köppen 1936), caracterizado como quente e úmido, com ausência de estação seca. A precipitação média anual para o município de Camacan é de 1.200 mm e a média mensal varia de 57 a 142 $\mathrm{mm}$ (i.e. dados coletados na Estação CEPLAC). A temperatura média anual é de $23^{\circ}$ a $24^{\circ} \mathrm{C}$ e a evapotranspiração potencial total é de 1.200 a $1.300 \mathrm{~mm} / \mathrm{ano}$, sendo a umidade relativa do ar em torno de $80 \%$ (Amorim et al. 2009). Ainda não existem informações sobre precipitação e temperatura, coletados de acordo com protocolos mundiais, específicas para a RPPN Serra Bonita, contudo dados pluviométricos foram coletados em alguns meses na área, registrado valores bem mais elevados, confirmando que há uma variação climática acentuada entre o topo da montanha e as áreas mais baixas. A exemplo disso, dados coletados no município de Camacan (ca. 150 m s.n.m.), nos meses de julho e agosto nos últimos dez anos obteve média de 79,1 e 64,9 mm, respectivamente, e nestes mesmos meses no ano de 2010, foram documentados 301 e $211 \mathrm{~mm}$ respectivamente, no alto da montanha (ca. $800 \mathrm{~m}$ s.n.m.). 

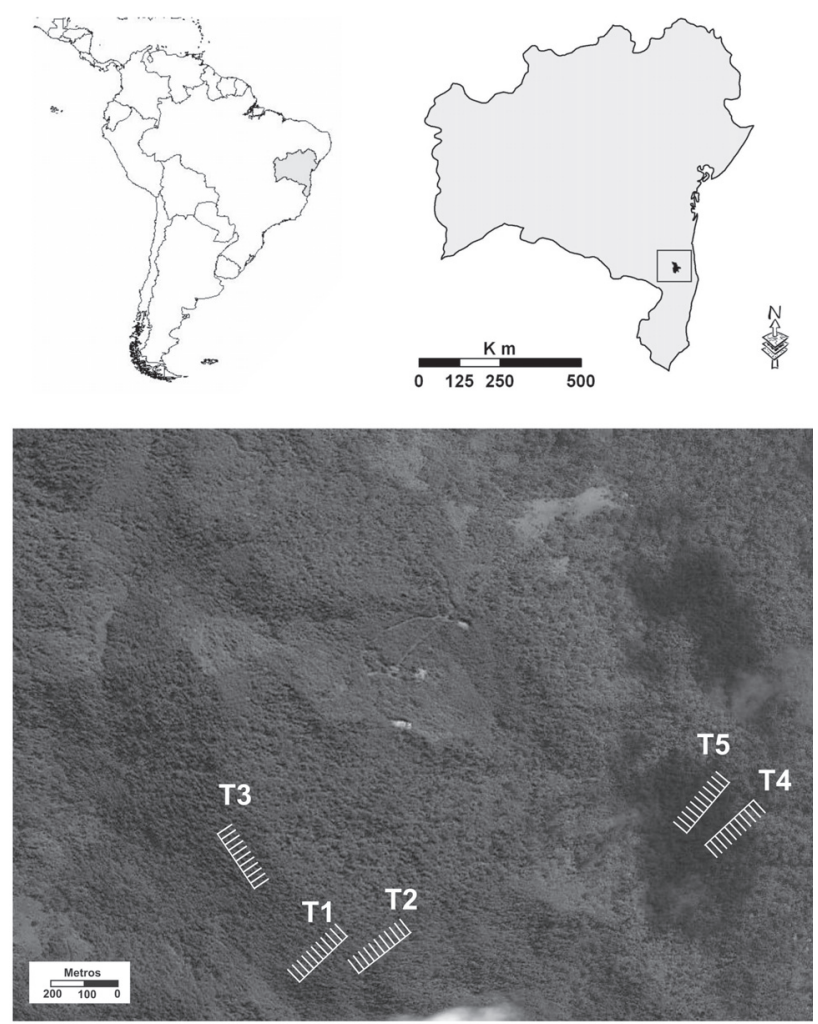

Figura 1. Localização geográfica da RPPN Serra Bonita, Bahia, Brasil indicando a disposição dos cinco plots amostrados (T1 - plot 1, T2 - plot 2,...).

\section{Amostragem}

Para a amostragem da vegetação lenhosa foi utilizado o método de parcelas de Gentry (1982) e que foi também aplicado por Martini et al. (2007) no sul da Bahia, que totaliza nesse estudo 0,5 ha. Foram alocados cinco plots de 0,1 ha cada (Fig. 1), sendo três a ca. 850 m s.n.m. (área 1) e dois a ca. $500 \mathrm{~m}$ s.n.m. (área 2). Foram mensuradas as alturas, marcadas e coletadas todas as plantas vasculares lenhosas vivas com DAP $\geq 2,5 \mathrm{~cm}$, inclusive lianas e bambus totalizando 1604 amostras. Todo o material foi herborizado seguindo metodologia usual descrita por Mori et al. (1989) e amostras testemunho de cada espécie foram incorporadas nos herbários CEPEC e HUEFS. O material foi identificado com base em literatura especializada, em destaque Gentry (1996), bem como por comparação com material previamente depositado no CEPEC onde existe uma coleção da área de estudo com coletas desde 2004 e que serviram de base para as investigações prévias, apresentadas por Amorim et al. (2009). Sempre que possível as determinações foram feitas e/ou confirmadas com a colaboração de especialistas (ver agradecimentos). A lista de espécies foi organizada seguindo as famílias reconhecidas por APG III (2009).

Para a análise da composição física e química do solo foram coletadas amostras a 0-20 e 20-40 cm de profundidade em três subunidades amostrais de cada plot selecionados de maneira aleatória. Todas as amostras de solo da mesma camada (0-20cm e 20-40) e mesmo plot foram homogenei- zadas, totalizando 10 amostras para a área total. As amostras foram enviadas ao laboratório de solos do Centro de Pesquisas do Cacau - CEPEC para análises de propriedades físicas (textura e condutividade) e químicas ( $\mathrm{pH}, \mathrm{Ca}, \mathrm{P}, \mathrm{N}$ e Mg).

\section{Análise dos dados}

Para comparar as diferenças na diversidade entre as duas áreas, utilizaram-se perfis de diversidade com a série de Rényi (Peet 1974), realizando os cálculos em três etapas, onde em cada uma retirava-se uma amostra excedente da área a $800 \mathrm{~m}$ s.n.m. com o intuito de padronizar o esforço amostral. Este método auxilia na solução do problema da falta de critério na escolha dos índices de diversidade (Melo 2008).

Para cada espécie calculou-se os parâmetros de densidade e dominância relativa e, para cada área amostrada, foram calculadas a densidade e área basal por hectare, os principais parâmetros fitossociológicos (Mueller-Dombois \& Ellenberg 1974), além do índice de Shannon ( $\left.\mathrm{H}^{\prime}\right)$ com base no logaritmo neperiano. Na comparação da composição florística das comunidades nas duas áreas, foi calculada a similaridade entre cada plot utilizando-se o coeficiente de Morisita (Magurran 1988) e elaborado um dendrograma através do método de agrupamento por média de grupo (UPGMA) com base em 10.000 réplicas de bootstrap.

Segundo Gotteli \& Colwell (2001) a comparação entre dados brutos geralmente produzem resultados enganosos. Assim, a riqueza das comunidades de cada altitude, bem como a de outros inventários, foi comparada através do método de rarefação baseadas em número de indivíduos com o intuito de padronizar o esforço amostral nas duas áreas (Gotteli \& Colwell 2001; Magurran 2004). Todos os cálculos foram realizados com auxílio dos softwares PAST (Hammer et al. 2001) e FITOPAC 1.6 (Shepherd 1995).

\section{Resultados e discussão}

Foram reconhecidas na comunidade 354 espécies de plantas vasculares lenhosas distribuídas em 68 famílias (Tab. 1). Foram identificados $71 \%$ dos táxons ao nível específico, 19\% ao nível de gênero e do restante conhece-se apenas a família. O índice de Shannon (H') foi de 4,818 nats./ ind. na área 1 e 4,897 nats./ind. na área 2. E estes valores ficaram acima da maior parte dos valores encontrados em floresta ombrófila de algumas regiões do Brasil, sendo que o H' apresentou valor muito próximo aos calculados (com mesma base logarítmica do presente estudo) a partir dos resultados encontrados em Martini et al. (2007). Os valores de H'aqui encontrados estão entre os maiores já observados em levantamentos em comunidades arbóreas citadas na literatura. A equabilidade foi de 0,890 na área 1 e 0,921 na área 2, o que indica uma heterogeneidade alta, sem dominância acentuada de qualquer espécie em ambas as áreas.

Myrtaceae, Fabaceae, Rubiaceae e Lauraceae apresentaram respectivamente, as maiores riquezas específicas (Fig. 
2). Essas famílias, comumente são reportadas como as mais ricas em floresta ombrófila densa (Tabarelli \& Mantovani 1999; Oliveira-Filho \& Fontes 2000; Moreno et al. 2003; Guilherme et al. 2004; Thomas et al. 2008; 2009) onde Myrtaceae é comumente referida com destaque tanto em regiões Submontanas (Mori et al. 1983; Martini et al. 2007; Thomas et al. 2008) como em altitudes elevadas (Meireles et al. 2008; Thomas et al. 2009).

Entre as famílias com maior IVI estão as mesmas citadas com maior riqueza. Entretanto, aparece entre elas Nyctaginaceae devido ao fato de possuir alta dominância relativa, ou seja, apresentou indivíduos com grandes diâmetros. Do total de famílias, dez alcançaram os maiores valores de IVI representando assim $56,17 \%$. Fabaceae, por exemplo, apesar de apresentar densidade relativa abaixo de outras famílias, possuiu um alto valor de dominância e frequência relativa, resultando no maior IVI. Isto foi consequência da distribuição relativamente uniforme de indivíduos de grande porte. Ao contrário, Rubiaceae apresentou baixa dominância relativa, sendo a alta densidade o principal fator que a levou ao segundo maior valor de IVI.

Mais da metade do total de espécies encontradas (55\%) foi representado por dez famílias contrastando com um elevado número de famílias (24) que apresentaram uma só espécie. Registrou-se pela primeira vez na RPPN Serra Bonita, a ocorrência de Burseraceae e Combretaceae (duas e três spp. respectivamente). Amorim et al. (2009) sugeriram a ausência dessas famílias em remanescentes de floresta Montana no sul da Bahia, por tratarem-se de grupos com ocorrência preferencial em formações Submontanas, como já apontado nos estudos de Martini et al. (2007) e Amorim et al. (2008). No entanto, a não detecção dessas famílias, provavelmente se deu pelo fato de estarem representadas nessas áreas unicamente por espécies arbóreas, dificultando assim a sua documentação.

A altura média das árvores encontrada na RPPN Serra Bonita foi de $8,3 \mathrm{~m} \pm 6$. A floresta estudada apresenta um dossel denso composto por espécies com alturas entre 15 e $25 \mathrm{~m}$, apresentando uma composição variada, principalmente em relação à altitude sendo que, dentre estas, observa-se predominantemente na Área 1 Allophylus petiolulatus, Ecclinusa ramiflora, Eugenia schotiana, Inga capitata e Ocotea aciphylla. Enquanto isso, na Área 2 tem-se Cecropia glaziovii, Helicostylis tomentosa e Ixora sp01. Em contraposição, Tapirira guianensis foi uma das poucas espécies que se apresentou abundante tanto no dossel como no sub-bosque nas duas áreas.

É comum observar a presença de espécies emergentes ultrapassando os $30 \mathrm{~m}$, onde se pode citar Arapatiella psilophylla, Caryocar edule, Copaifera trapaezifolia, Garcinia gardneriana, Inga vera, Lamanonia ternata, Lecythis pisonis, Ocotea daphinifolia e Vochysia riedeliana. No sub-bosque Rubiaceae se sobressaiu, representada por muitas espécies arbustivas de pequeno porte, sendo Psychotria o gênero mais abundante. Além dessa, Piperaceae também foi abundante com destaque para Piper richardifolium. Ocorreram também, em menor abundância, indivíduos jovens de espécies comumente encontradas nos estratos superiores.

Em relação às classes de diâmetros, mais de $70 \%$ dos indivíduos na RPPN Serra Bonita apresentaram diâmetros de até $10 \mathrm{~cm}$, sendo que a maior concentração (40\%) se deu na classe entre 2,5 e $5 \mathrm{~cm}$ (Fig. 3). O elevado número de indivíduos nas classes iniciais sugere que esta comunidade é constituída por muitos indivíduos jovens, e encontra-se em crescimento eficiente, conforme sugerido por Maragon et al. (2008). A distribuição dos indivíduos nas classes de diâmetro formando uma série completa (i.e. sem interrupção) é indicativa de uma comunidade em equilíbrio, que em geral apresenta grande produção de sementes, germinação satisfatória e taxa de mortalidade decrescente nas idades mais avançadas (Pereira-Silva 2004).

Quando analisados os plots por áreas associadas com a altitude (i.e. área $1 \mathrm{a}$ ca. $800 \mathrm{~m}$ s.n.m., formada por T1, T2 e T3 e área 2 a ca. 500 m s.n.m., formada por T4 e T5), encontra-se 55 famílias em cada trecho, onde 42 mostram-se em comum. A ordenação decrescente em riqueza se mantém entre Myrtaceae, Fabaceae, Rubiaceae e Lauraceae ainda que, com sutis diferenças entre as áreas (Fig. 2). A substituição parcial de famílias fica visualizada em Melastomataceae e Sapotaceae que, quando comparadas entre si, mostram evidentes modificações no número de espécies (Fig. 2). Alteração na riqueza ao longo de um gradiente altitudinal foi percebida nos estudos de Moreno et al. (2003) e em Meireles et al. (2008), ambos realizados em florestas úmidas no sudeste brasileiro. Oliveira-Filho \& Fontes (2000) desta-

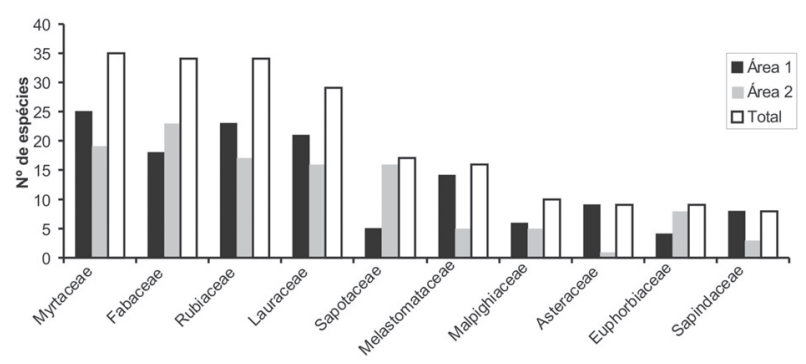

Figura 2. Número de espécies das 10 famílias mais ricas na RPPN Serra Bonita, Bahia, Brasil.

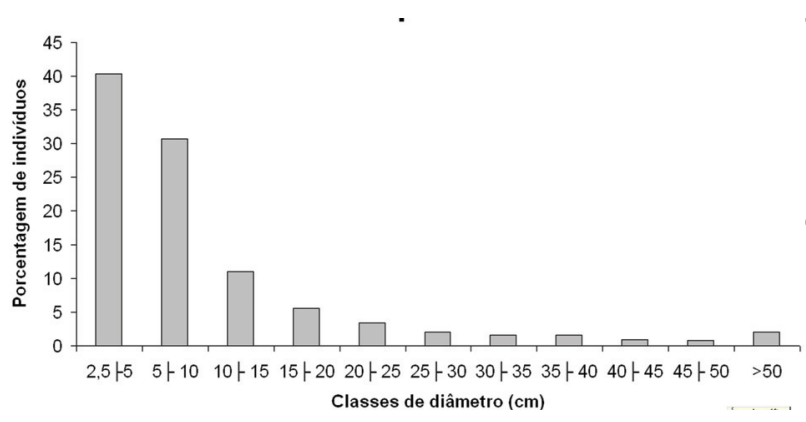

Figura 3. Distribuição das freqüências nas classes de diâmetro $(\mathrm{cm})$ na RPPN Serra Bonita, Bahia, Brasil. 

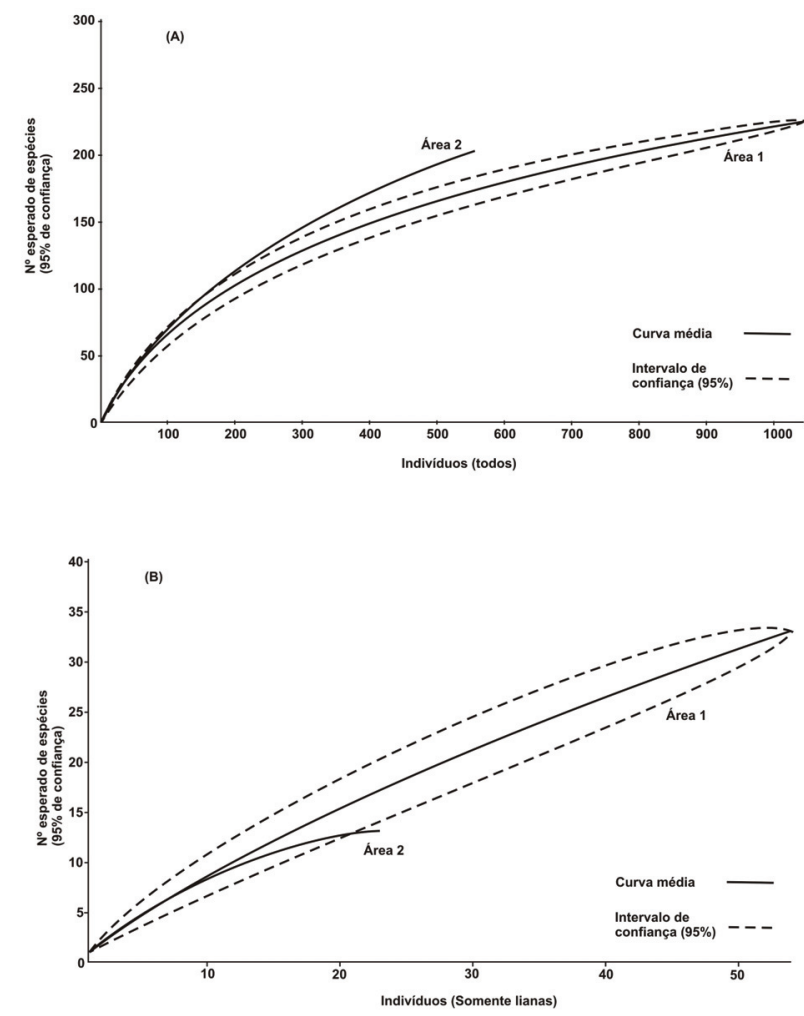

Figura 4. Curvas de rarefação. A e B ilustram as áreas na RPPN Serra Bonita, Bahia, Brasil. A inclui todos os indivíduos, B apenas lianas.

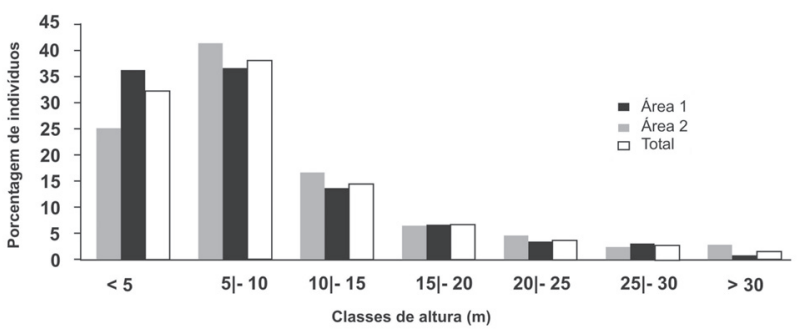

Figura 5. Distribuição das freqüências nas classes de diâmetro $(\mathrm{cm})$ na RPPN Serra Bonita, Bahia, Brasil.

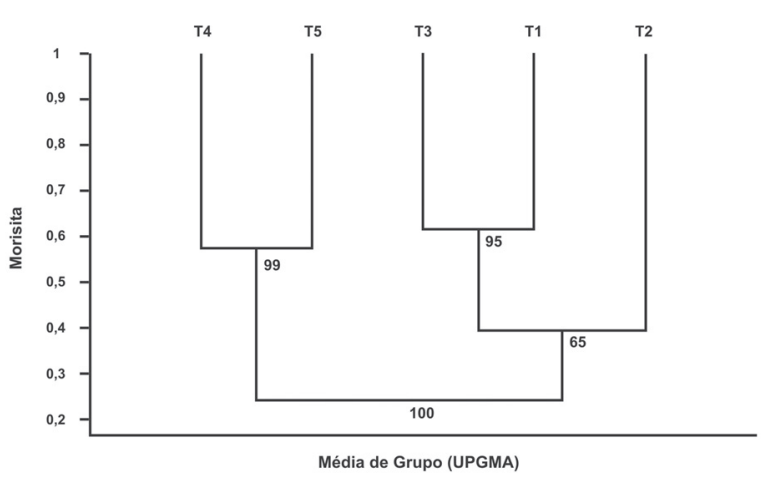

Figura 6. Dendrograma de similaridade de espécies para os plots (T1 a T3) da área 1 (800 m s.n.m.) e os plots (T4 e T5) da área 2 (500 m s.n.m.) na RPPN Serra Bonita, Bahia, Brasil, a partir do coeficiente de Morisita com método de agrupamento UPGMA. Os números internos se referem aos valores de bootstrap.
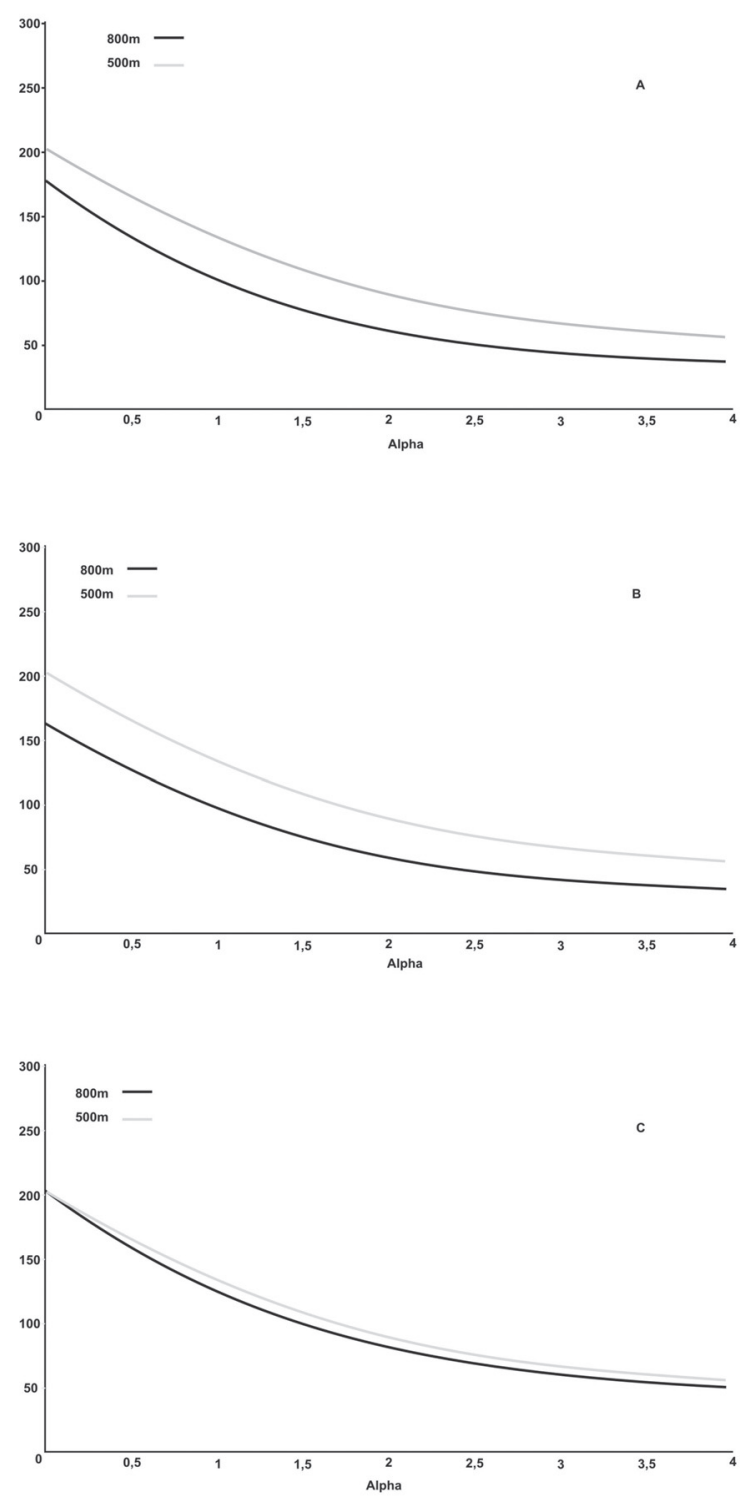

Figura 7. Perfis de diversidade para áreas amostradas em duas comunidades, na RPPN Serra Bonita, Bahia, Brasil usando a Série de Réyni. A- excluindo o T1, B- excluindo o T2 e C- excluindo o T3.

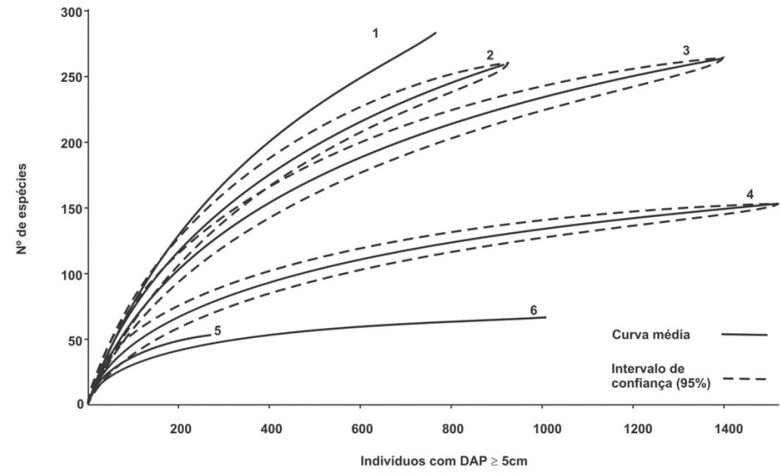

Figura 8. Curvas de rarefação utilizando somente indivíduos com DAP $\geq 5 \mathrm{~cm}$ em uma comparação com outros inventários: 1- Serra do Conduru - BA (Martini et al. 2007), 2-Serra Bonita-BA (Presente estudo), 3- Serra do Teimoso-BA (Thomas et al. 2009), 4- Borborema-PE (Ferraz \& Rodal 2006), 5- Imbé-RJ (Moreno et al. 2003) e 6- Mantiqueira-MG (Meireles et al. 2008). 
Tabela 1. Lista de espécies na RPPN Serra Bonita, Bahia, Brasil e respectivos parâmetros, em ordem alfabética. (NI = número total de indivíduos; DeR = densidade relativa; DoR = dominância relativa)

\begin{tabular}{|c|c|c|c|c|c|c|c|}
\hline Família & Espécies & $\begin{array}{c}500 \mathrm{~m} \\
\mathrm{NI}\end{array}$ & $\begin{array}{c}500 \mathrm{~m} \\
\mathrm{DeR}\end{array}$ & $\begin{array}{c}500 \mathrm{~m} \\
\text { DoR }\end{array}$ & $\begin{array}{c}800 \mathrm{~m} \\
\mathrm{NI}\end{array}$ & $\begin{array}{c}800 m \\
\text { DeR }\end{array}$ & $\begin{array}{c}800 \mathrm{~m} \\
\text { DoR }\end{array}$ \\
\hline Anacardiaceae & Tapirira guianensis Aubl. & 9 & 1,61 & 1,52 & 24 & 2,29 & 2,38 \\
\hline Anacardiaceae & Thyrsodium spruceanum Benth. & 3 & 0,54 & 0,42 & - & - & - \\
\hline Annonaceae & Annonaceae sp01 & 2 & 0,36 & 1,19 & - & - & - \\
\hline Annonaceae & Annona cacans Warm. & 1 & 0,18 & 0,17 & 1 & 0,1 & 0,2 \\
\hline Annonaceae & Annona dolabripetala Raddi & 1 & 0,18 & 0,22 & 3 & 0,29 & 0,46 \\
\hline Annonaceae & Guatteria australis A.St.-Hil. & - & - & - & 1 & 0,1 & 0,07 \\
\hline Annonaceae & Guatteria pogonopus Mart. & - & - & - & 5 & 0,48 & 0,3 \\
\hline Annonaceae & Unonopsis bahiensis Maas \& Orava & 1 & 0,18 & 0 & - & - & - \\
\hline Annonaceae & Xylopia sericea A.St.-Hil. & 1 & 0,18 & 0,03 & - & - & - \\
\hline Apocynaceae & Aspidosperma sp01 & 1 & 0,18 & 0,01 & - & - & - \\
\hline Apocynaceae & Lacmellea pauciflora (Kuhlm.) Markgr. & 1 & 0,18 & 0,2 & - & - & - \\
\hline Apocynaceae & Peltastes peltatus (Vell.) Woodson & - & - & - & 1 & 0,1 & 0,62 \\
\hline Apocynaceae & Rauvolfia grandiflora Mart. ex A.DC. & 1 & 0,18 & 0,01 & - & - & - \\
\hline Apocynaceae & Rauvolfia sp01 & 1 & 0,18 & 0,05 & - & - & - \\
\hline Apocynaceae & Tabernaemontana linkii A.DC. & 1 & 0,18 & 0,57 & - & - & - \\
\hline Aquifoliaceae & Ilex paraguariensis A.St.-Hil. & 1 & 0,18 & 0,17 & 3 & 0,29 & 0,05 \\
\hline Araliaceae & Dendropanax bahiensis Fiaschi & - & - & - & 1 & 0,1 & 0,03 \\
\hline Araliaceae & Schefflera angustissima (Marchal) Frodin & - & - & - & 1 & 0,1 & 0 \\
\hline Arecaceae & Euterpe edulis Mart. & 10 & 1,79 & 1,64 & 25 & 2,39 & 1,56 \\
\hline Arecaceae & Geonoma gamiova Barb.Rodr. & - & - & - & 23 & 2,2 & 0,7 \\
\hline Asteraceae & Heterocondylus vitalbae (DC.) R.M.King \& H.Rob. & - & - & - & 1 & 0,1 & 0 \\
\hline Asteraceae & Mikania biformis DC. & - & - & - & 1 & 0,1 & 0 \\
\hline Asteraceae & Mikania callineura Sch.Bip. ex Baker & - & - & - & 4 & 0,38 & 0,05 \\
\hline Asteraceae & Mikania candolleana Gardner & - & - & - & 1 & 0,1 & 0 \\
\hline Asteraceae & Mikania mattos-silvae R.M.King \& H.Rob. & 2 & 0,36 & 0,03 & 9 & 0,86 & 0,06 \\
\hline Asteraceae & Mikania trinervis Hook. \& Arn. & - & - & - & 1 & 0,1 & 0,01 \\
\hline Asteraceae & Piptocarpha ramiflora (Spreng.) Baker & - & - & - & 1 & 0,1 & 0,01 \\
\hline Asteraceae & Piptocarpha sp01 & - & - & - & 1 & 0,1 & 0,02 \\
\hline Asteraceae & Vernonanthura diffusa (Less.) H.Rob. & - & - & - & 5 & 0,48 & 0,29 \\
\hline Bignoniaceae & Bignoniaceae sp01 & 2 & 0,36 & 0,04 & - & - & - \\
\hline Bignoniaceae & Bignoniaceae sp02 & 2 & 0,36 & 0,01 & - & - & - \\
\hline Bignoniaceae & Amphilophium crucigerum (L.) L.G.Lohmann & - & - & - & 2 & 0,19 & 0,02 \\
\hline Bignoniaceae & Handroanthus heptaphyllus (Vell.) Mattos & 4 & 0,72 & 3,93 & 6 & 0,57 & 0,28 \\
\hline Bignoniaceae & Jacaranda puberula Cham. & 1 & 0,18 & 0 & - & - & - \\
\hline Bignoniaceae & Lundia cordata (Vell.) DC. & 2 & 0,36 & 0,03 & - & - & - \\
\hline Boraginaceae & Cordia sp01 & 2 & 0,36 & 0,21 & - & - & - \\
\hline Boraginaceae & Cordia sp02 & - & - & - & 1 & 0,1 & 0,17 \\
\hline Boraginaceae & Cordia sp03 & 1 & 0,18 & 0,01 & - & - & - \\
\hline Boraginaceae & Cordia trichoclada DC. & - & - & - & 1 & 0,1 & 0,01 \\
\hline Burseraceae & Crepidospermum atlanticum Daly & 2 & 0,36 & 0,04 & - & - & - \\
\hline Burseraceae & Protium aracouchini (Aubl.) Marchand & 6 & 1,08 & 0,16 & - & - & - \\
\hline Cardiopteridaceae & Citronella gongonha (Mart.) R.A.Howard & - & - & - & 1 & 0,1 & 0,04 \\
\hline Cardiopteridaceae & Citronella megaphylla (Miers) R.A.Howard & 1 & 0,18 & 0,29 & - & - & - \\
\hline
\end{tabular}


Tabela 1. Continuação.

\begin{tabular}{|c|c|c|c|c|c|c|c|}
\hline Família & Espécies & $\begin{array}{c}500 \mathrm{~m} \\
\text { NI }\end{array}$ & $\begin{array}{c}500 \mathrm{~m} \\
\text { DeR }\end{array}$ & $\begin{array}{c}500 \mathrm{~m} \\
\text { DoR }\end{array}$ & $\begin{array}{c}800 \mathrm{~m} \\
\text { NI }\end{array}$ & $\begin{array}{c}800 \mathrm{~m} \\
\text { DeR }\end{array}$ & $\begin{array}{c}800 \mathrm{~m} \\
\text { DoR }\end{array}$ \\
\hline Caricaceae & Jacaratia heptaphylla (Vell.) A.DC. & 4 & 0,72 & 0,36 & - & - & - \\
\hline Caryocaraceae & Caryocar edule Casar. & 1 & 0,18 & 5 & - & - & - \\
\hline Celastraceae & Cheiloclinium cognatum (Miers) A.C.Sm. & - & - & - & 4 & 0,38 & 1,19 \\
\hline Celastraceae & Maytenus brasiliensis Mart. & 5 & 0,9 & 0,14 & - & - & - \\
\hline Celastraceae & Salacia grandifolia (Mart. ex Schult.) G.Don & 5 & 0,9 & 0,26 & - & - & - \\
\hline Chrysobalanaceae & Couepia belemii Prance & - & - & - & 14 & 1,34 & 0,22 \\
\hline Chrysobalanaceae & Couepia monteclarensis Prance & - & - & - & 3 & 0,29 & 0,09 \\
\hline Chrysobalanaceae & Hirtella santosii Prance & 2 & 0,36 & 0,05 & - & - & - \\
\hline Chrysobalanaceae & Licania belemii Prance & 2 & 0,36 & 0,71 & - & - & - \\
\hline Chrysobalanaceae & Licania naviculistipula Prance & 2 & 0,36 & 0,05 & - & - & - \\
\hline Chrysobalanaceae & Licania sp01 & - & - & - & 3 & 0,29 & 0,42 \\
\hline Clethraceae & Clethra scabra Pers. & - & - & - & 1 & 0,1 & 0,32 \\
\hline Clethraceae & Clethra sp01 & - & - & - & 4 & 0,38 & 0,27 \\
\hline Clusiaceae & Garcinia gardneriana (Planch. \& Triana) Zappi & - & - & - & 10 & 0,96 & 1,62 \\
\hline Clusiaceae & Kielmeyera neglecta Saddi & - & - & - & 10 & 0,96 & 1,12 \\
\hline Clusiaceae & Tovomita sp01 & 2 & 0,36 & 0,01 & 10 & 0,96 & 0,07 \\
\hline Clusiaceae & Tovomita sp02 & - & - & - & 4 & 0,38 & 0,06 \\
\hline Clusiaceae & Tovomita choisyana Planch. \& Triana & 1 & 0,18 & 0 & - & - & - \\
\hline Combretaceae & Buchenavia hoehneana N.F.Mattos & 1 & 0,18 & 0,04 & - & - & - \\
\hline Combretaceae & Terminalia sp01 & - & - & - & 1 & 0,1 & 0,02 \\
\hline Combretaceae & Terminalia kuhlmannii Alwan \& Stace & 1 & 0,18 & 0,46 & - & - & - \\
\hline Cunoniaceae & Lamanonia ternata Vell. & 1 & 0,18 & 0,41 & 1 & 0,1 & 4,56 \\
\hline Cyatheaceae & Alsophila setosa Moric. & - & - & - & 1 & 0,1 & 0,01 \\
\hline Cyatheaceae & Cyathea corcovadensis (Raddi) Domin & 8 & 1,43 & 0,19 & - & - & - \\
\hline Cyatheaceae & Cyathea delgadii Sternb. & 1 & 0,18 & 0,03 & 18 & 1,72 & 1,21 \\
\hline Cyatheaceae & Cyathea glaziovii (Fée) Domin & - & - & - & 4 & 0,38 & 0,11 \\
\hline Cyatheaceae & Cyathea phalerata Mart. & - & - & - & 42 & 4,02 & 1,48 \\
\hline Cyatheaceae & Cyathea praecincta (Kunze) Domin & 4 & 0,72 & 0,09 & - & - & - \\
\hline Dichapetalaceae & Stephanopodium blanchetianum Baill. & 2 & 0,36 & 0,27 & 3 & 0,29 & 0,68 \\
\hline Ebenaceae & Diospyros apeibacarpos Raddi & - & - & - & 1 & 0,1 & 0,01 \\
\hline Elaeocarpaceae & Sloanea sp01 & 1 & 0,18 & 0,01 & - & - & - \\
\hline Elaeocarpaceae & Sloanea guianensis (Aubl.) Benth. & 5 & 0,9 & 0,6 & 4 & 0,38 & 0,07 \\
\hline Erythroxylaceae & Erythroxylum sp01 & 2 & 0,36 & 0,03 & - & - & - \\
\hline Erythroxylaceae & Erythroxylum compressum Peyr. & - & - & - & 5 & 0,48 & 0,07 \\
\hline Erythroxylaceae & Erythroxylum cuspidifolium Mart. & - & - & - & 2 & 0,19 & 0,01 \\
\hline Erythroxylaceae & Erythroxylum grandifolium Peyr. & - & - & - & 1 & 0,1 & 0,01 \\
\hline Euphorbiaceae & Euphorbiaceae sp01 & 2 & 0,36 & 0,02 & - & - & - \\
\hline Euphorbiaceae & Alchornea triplinervia (Spreng.) Müll.Arg. & - & - & - & 11 & 1,05 & 3,82 \\
\hline Euphorbiaceae & Aparisthmium cordatum (A.Juss.) Baill. & 8 & 1,43 & 0,36 & 1 & 0,1 & 0,01 \\
\hline Euphorbiaceae & Croton macrobothrys Baill. & 3 & 0,54 & 0,31 & - & - & - \\
\hline Euphorbiaceae & Glycydendron amazonicum Ducke & 1 & 0,18 & 0,25 & - & - & - \\
\hline Euphorbiaceae & Mabea brasiliensis Müll.Arg. & 7 & 1,25 & 0,33 & - & - & - \\
\hline Euphorbiaceae & Ophthalmoblapton pedunculare Müll.Arg. & 3 & 0,54 & 0,02 & 5 & 0,48 & 0,29 \\
\hline Euphorbiaceae & Senefeldera verticillata (Vell.) Croizat & 2 & 0,36 & 0,22 & - & - & - \\
\hline
\end{tabular}


Tabela 1. Continuação.

\begin{tabular}{|c|c|c|c|c|c|c|c|}
\hline Família & Espécies & $\begin{array}{c}500 \mathrm{~m} \\
\text { NI }\end{array}$ & $\begin{array}{c}500 \mathrm{~m} \\
\text { DeR }\end{array}$ & $\begin{array}{c}500 \mathrm{~m} \\
\text { DoR }\end{array}$ & $\begin{array}{c}800 \mathrm{~m} \\
\text { NI }\end{array}$ & $\begin{array}{c}800 \mathrm{~m} \\
\text { DeR }\end{array}$ & $\begin{array}{c}800 \mathrm{~m} \\
\text { DoR }\end{array}$ \\
\hline Euphorbiaceae & Tetrorchidium rubrivenium Poepp. \& Endl. & 2 & 0,36 & 1,58 & - & - & - \\
\hline Fabaceae & Fabaceae sp01 & 2 & 0,36 & 0,02 & - & - & - \\
\hline Fabaceae & Fabaceae sp02 & 2 & 0,36 & 0,06 & 2 & 0,19 & 0,04 \\
\hline Fabaceae & Andira sp01 & 2 & 0,36 & 0,14 & - & - & - \\
\hline Fabaceae & Arapatiella psilophylla (Harms) R.S.Cowan & 6 & 1,08 & 7,53 & - & - & - \\
\hline Fabaceae & Bauhinia sp01 & - & - & - & 2 & 0,19 & 1,64 \\
\hline Fabaceae & Copaifera trapaezifolia Hayne & - & - & - & 9 & 0,86 & 1,47 \\
\hline Fabaceae & Dalbergia frutescens (Vell.) Britton & 2 & 0,36 & 0,03 & - & - & - \\
\hline Fabaceae & Dioclea edulis Kuhlm. & - & - & - & 3 & 0,29 & 0,03 \\
\hline Fabaceae & Exostyles venusta Schott ex Spreng. & 2 & 0,36 & 0,03 & - & - & - \\
\hline Fabaceae & Hymenolobium janeirense Kuhlm. & - & - & - & 1 & 0,1 & 0,11 \\
\hline Fabaceae & Inga sp01 & 1 & 0,18 & 0,14 & - & - & - \\
\hline Fabaceae & Inga sp02 & 1 & 0,18 & 0 & - & - & - \\
\hline Fabaceae & Inga capitata Desv. & 1 & 0,18 & 0,01 & 20 & 1,91 & 0,88 \\
\hline Fabaceae & Inga grazielae (Vinha) T.D.Penn. & - & - & - & 5 & 0,48 & 2,23 \\
\hline Fabaceae & Inga marginata Willd. & 1 & 0,18 & 0,04 & - & - & - \\
\hline Fabaceae & Inga mendoncaei Harms & - & - & - & 1 & 0,1 & 0,01 \\
\hline Fabaceae & Inga striata Benth. & 1 & 0,18 & 0 & - & - & - \\
\hline Fabaceae & Inga tenuis (Vell.) Mart. & 3 & 0,54 & 0,25 & 4 & 0,38 & 0,03 \\
\hline Fabaceae & Inga thibaudiana DC. & 2 & 0,36 & 0,17 & 1 & 0,1 & 0,02 \\
\hline Fabaceae & Inga vera Willd. & - & - & - & 9 & 0,86 & 2,3 \\
\hline Fabaceae & Machaerium sp01 & - & - & - & 2 & 0,19 & 0,06 \\
\hline Fabaceae & Machaerium sp02 & 4 & 0,72 & 6,72 & - & - & - \\
\hline Fabaceae & Machaerium nyctitans (Vell.) Benth. & 1 & 0,18 & 0,25 & - & - & - \\
\hline Fabaceae & Machaerium reticulatum (Poir.) Pers. & - & - & - & 3 & 0,29 & 0,04 \\
\hline Fabaceae & Machaerium stipitatum (DC.) Vogel & 1 & 0,18 & 0,01 & - & - & - \\
\hline Fabaceae & Machaerium uncinatum (Vell.) Benth. & 1 & 0,18 & 0,01 & 1 & 0,1 & 0,01 \\
\hline Fabaceae & Macrolobium latifolium Vogel & 6 & 1,08 & 2,69 & - & - & - \\
\hline Fabaceae & Ormosia fastigiata Tul. & - & - & - & 2 & 0,19 & 0,04 \\
\hline Fabaceae & Senegalia martiusiana (Steud.) Seigler \& Ebinger & - & - & - & 1 & 0,1 & 0,01 \\
\hline Fabaceae & Swartzia oblata R.S.Cowan & 1 & 0,18 & 0 & - & - & - \\
\hline Fabaceae & Swartzia simplex (Sw.) Spreng. var. continentalis Urb. & 1 & 0,18 & 0,01 & - & - & - \\
\hline Fabaceae & Tachigali paratyensis (Vell.) H.C.Lima & 5 & 0,9 & 1,94 & 2 & 0,19 & 0,07 \\
\hline Fabaceae & Vataireopsis araroba (Aguiar) Ducke & 2 & 0,36 & 0,01 & - & - & - \\
\hline Fabaceae & Zollernia latifolia Benth. & 2 & 0,36 & 0,02 & 1 & 0,1 & 0,01 \\
\hline Icacinaceae & Leretia sp & - & - & - & 1 & 0,1 & 0,02 \\
\hline Indeterminada & Indeterminada sp01 & 2 & 0,36 & 0,08 & - & - & - \\
\hline Indeterminada & Indeterminada sp02 & 1 & 0,18 & 0,02 & - & - & - \\
\hline Lauraceae & Lauraceae sp01 & - & - & - & 2 & 0,19 & 0,02 \\
\hline Lauraceae & Lauraceae sp02 & - & - & - & 2 & 0,19 & 0,02 \\
\hline Lauraceae & Lauraceae sp03 & - & - & - & 1 & 0,1 & 0 \\
\hline Lauraceae & Lauraceae sp04 & 1 & 0,18 & 0,01 & - & - & - \\
\hline Lauraceae & Lauraceae sp05 & 2 & 0,36 & 0,19 & - & - & - \\
\hline
\end{tabular}


Tabela 1. Continuação.

\begin{tabular}{|c|c|c|c|c|c|c|c|}
\hline Família & Espécies & $\begin{array}{l}500 \mathrm{~m} \\
\mathrm{NI}\end{array}$ & $\begin{array}{c}500 \mathrm{~m} \\
\text { DeR }\end{array}$ & $\begin{array}{l}500 \mathrm{~m} \\
\mathrm{DoR}\end{array}$ & $\begin{array}{l}800 \mathrm{~m} \\
\mathrm{NI}\end{array}$ & $\begin{array}{c}800 \mathrm{~m} \\
\mathrm{DeR}\end{array}$ & $\begin{array}{c}800 \mathrm{~m} \\
\text { DoR }\end{array}$ \\
\hline Lauraceae & Lauraceae sp06 & 1 & 0,18 & 0,06 & 6 & 0,57 & 0,52 \\
\hline Lauraceae & Lauraceae sp07 & 1 & 0,18 & 0,01 & - & - & - \\
\hline Lauraceae & Lauraceae sp08 & - & - & - & 1 & 0,1 & 0,12 \\
\hline Lauraceae & Aiouea laevis (Nees ex Mart.) Kosterm. & 1 & 0,18 & 0,01 & - & - & - \\
\hline Lauraceae & Aniba intermedia (Meisn.) Mez & 1 & 0,18 & 0,16 & - & - & - \\
\hline Lauraceae & Aniba sp01 & 1 & 0,18 & 0,07 & 10 & 0,96 & 1,07 \\
\hline Lauraceae & Licaria bahiana H. Kurz & 1 & 0,18 & 0,01 & 3 & 0,29 & 0,09 \\
\hline Lauraceae & Nectandra membranacea (Sw.) Griseb. & 2 & 0,36 & 0,16 & 3 & 0,29 & 0,06 \\
\hline Lauraceae & Nectandra sp01 & 1 & 0,18 & 0 & - & - & - \\
\hline Lauraceae & Ocotea sp01 & 1 & 0,18 & 0 & - & - & - \\
\hline Lauraceae & Ocotea sp02 & 1 & 0,18 & 0,01 & 1 & 0,1 & 0,01 \\
\hline Lauraceae & Ocotea sp03 & - & - & - & 1 & 0,1 & 0,01 \\
\hline Lauraceae & Ocotea sp04 & - & - & - & 1 & 0,1 & 0 \\
\hline Lauraceae & Ocotea sp05 & - & - & - & 1 & 0,1 & 0,14 \\
\hline Lauraceae & Ocotea sp06 & - & - & - & 1 & 0,1 & 0,06 \\
\hline Lauraceae & Ocotea sp07 & - & - & - & 1 & 0,1 & 0,01 \\
\hline Lauraceae & Ocotea aciphylla (Nees) Mez & 4 & 0,72 & 0,61 & 9 & 0,86 & 1,98 \\
\hline Lauraceae & Ocotea canaliculata (Rich.) Mez & 2 & 0,36 & 0,11 & - & - & - \\
\hline Lauraceae & Ocotea cernua (Nees) Mez & - & - & - & 5 & 0,48 & 0,94 \\
\hline Lauraceae & Ocotea corymbosa (Meisn.) Mez & - & - & - & 1 & 0,1 & 0,03 \\
\hline Lauraceae & Ocotea daphnifolia (Meisn.) Mez & 3 & 0,54 & 3,95 & 8 & 0,76 & 0,5 \\
\hline Lauraceae & Ocotea divaricata (Nees) Mez & 1 & 0,18 & 0,11 & 4 & 0,38 & 0,18 \\
\hline Lauraceae & Ocotea leucoxylon (Sw.) Laness. & - & - & - & 4 & 0,38 & 0,1 \\
\hline Lauraceae & Persea caesia Meisn. & - & - & - & 4 & 0,38 & 0,23 \\
\hline Lecythidaceae & Eschweilera ovata (Cambess.) Mart. ex Miers & 1 & 0,18 & 0,02 & - & - & - \\
\hline Lecythidaceae & Lecythis pisonis Cambess. & 5 & 0,9 & 0,89 & - & - & - \\
\hline Loganiaceae & Strychnos atlantica Krukoff \& Barneby & 2 & 0,36 & 0,09 & - & - & - \\
\hline Malpighiaceae & Malpighiaceae sp01 & 1 & 0,18 & 0,01 & - & - & - \\
\hline Malpighiaceae & Malpighiaceae sp02 & 1 & 0,18 & 0,07 & - & - & - \\
\hline Malpighiaceae & Banisteriopsis membranifolia (A.Juss.) B.Gates & 4 & 0,72 & 0,15 & - & - & - \\
\hline Malpighiaceae & Byrsonima sp01 & - & - & - & 1 & 0,1 & 0,01 \\
\hline Malpighiaceae & Byrsonima sp02 & 1 & 0,18 & 0,31 & - & - & - \\
\hline Malpighiaceae & Byrsonima laevigata (Poir.) DC. & 1 & 0,18 & 0,02 & 1 & 0,1 & 0,01 \\
\hline Malpighiaceae & $\begin{array}{l}\text { Diplopterys patula (B.Gates) } \\
\text { W.R.Anderson \& C.C.Davis }\end{array}$ & - & - & - & 1 & 0,1 & 0,02 \\
\hline Malpighiaceae & Heteropterys bullata Amorim & - & - & - & 2 & 0,19 & 0,01 \\
\hline Malpighiaceae & Heteropterys nitida (Lam.) DC. & - & - & - & 2 & 0,19 & 0,04 \\
\hline Malpighiaceae & Heteropterys nordestina Amorim & - & - & - & 1 & 0,1 & 0,01 \\
\hline Malvaceae & Eriotheca macrophylla (K.Schum.) A.Robyns & 9 & 1,61 & 1,23 & - & - & - \\
\hline Malvaceae & Pavonia calyculosa A.St.-Hil. \& Naudin & 1 & 0,18 & 0,36 & - & - & - \\
\hline Malvaceae & Sterculia excelsa Mart. & 1 & 0,18 & 0,12 & - & - & - \\
\hline Marantaceae & Marantaceae sp01 & 1 & 0,18 & 0,01 & - & - & - \\
\hline Melastomataceae & Graffenrieda intermedia Triana & - & - & - & 1 & 0,1 & 0,01 \\
\hline Melastomataceae & Leandra clidemioides (Naudin) Wurdack & 6 & 1,08 & 0,05 & - & - & - \\
\hline
\end{tabular}


Tabela 1. Continuação.

\begin{tabular}{|c|c|c|c|c|c|c|c|}
\hline Família & Espécies & $\begin{array}{l}500 \mathrm{~m} \\
\mathrm{NI}\end{array}$ & $\begin{array}{c}500 \mathrm{~m} \\
\mathrm{DeR}\end{array}$ & $\begin{array}{c}500 \mathrm{~m} \\
\text { DoR }\end{array}$ & $\begin{array}{l}800 \mathrm{~m} \\
\mathrm{NI}\end{array}$ & $\begin{array}{c}800 \mathrm{~m} \\
\mathrm{DeR}\end{array}$ & $\begin{array}{l}800 \mathrm{~m} \\
\text { DoR }\end{array}$ \\
\hline Melastomataceae & Leandra cuneata (Mart.) Cogn. & 1 & 0,18 & 0,01 & 1 & 0,1 & 0,01 \\
\hline Melastomataceae & Leandra dasytricha (A.Gray) Cogn. & - & - & - & 1 & 0,1 & 0,01 \\
\hline Melastomataceae & Leandra melastomoides Raddi & - & - & - & 5 & 0,48 & 0,03 \\
\hline Melastomataceae & Meriania tetramera Wurdack & - & - & - & 6 & 0,57 & 0,2 \\
\hline Melastomataceae & Miconia budlejoides Triana & 1 & 0,18 & 0,01 & 9 & 0,86 & 0,15 \\
\hline Melastomataceae & Miconia doriana Cogn. & - & - & - & 1 & 0,1 & 0,01 \\
\hline Melastomataceae & Miconia fasciculata Gardner & - & - & - & 4 & 0,38 & 3,11 \\
\hline Melastomataceae & Miconia formosa Cogn. & - & - & - & 4 & 0,38 & 0,04 \\
\hline Melastomataceae & Miconia minutiflora (Bonpl.) DC. & - & - & - & 4 & 0,38 & 0,07 \\
\hline Melastomataceae & Miconia mirabilis (Aubl.) L.O.Williams & 2 & 0,36 & 1,62 & 8 & 0,76 & 0,68 \\
\hline Melastomataceae & Miconia pusilliflora (DC.) Naudin & - & - & - & 11 & 1,05 & 0,41 \\
\hline Melastomataceae & Miconia tristis Spring & - & - & - & 1 & 0,1 & 0,01 \\
\hline Melastomataceae & Mouriri bahiensis Morley & 3 & 0,54 & 0,54 & - & - & - \\
\hline Melastomataceae & Tibouchina fissinervia (DC.) Cogn. & - & - & - & 2 & 0,19 & 0,41 \\
\hline Meliaceae & Cabralea canjerana (Vell.) Mart. & 1 & 0,18 & 0,01 & 2 & 0,19 & 0,01 \\
\hline Meliaceae & Guarea kunthiana A.Juss. & 1 & 0,18 & 0,01 & - & - & - \\
\hline Meliaceae & Guarea macrophylla Vahl. & 3 & 0,54 & 0,08 & 1 & 0,1 & 0,04 \\
\hline Meliaceae & Trichilia lepidota Mart. & 1 & 0,18 & 0,01 & - & - & - \\
\hline Meliaceae & Trichilia quadrijuga H.B.K. & - & - & - & 10 & 0,96 & 3,25 \\
\hline Meliaceae & Trichilia silvatica C.DC. & 2 & 0,36 & 0,01 & - & - & - \\
\hline Menispermaceae & Anomospermum reticulatum (Mart.) Eichler & 1 & 0,18 & 0,08 & - & - & - \\
\hline Menispermaceae & Chondrodendron platyphyllum (A.St.-Hil.) Miers & - & - & - & 1 & 0,1 & 0 \\
\hline Menispermaceae & Curarea crassa Barneby & 2 & 0,36 & 0,05 & 1 & 0,1 & 0,02 \\
\hline Monimiaceae & Mollinedia gilgiana Perkins & - & - & - & 7 & 0,67 & 0,3 \\
\hline Monimiaceae & Mollinedia oligantha Perkins & - & - & - & 7 & 0,67 & 0,13 \\
\hline Monimiaceae & Mollinedia ovata Ruiz \& Pav. & 3 & 0,54 & 0,47 & 2 & 0,19 & 0,01 \\
\hline Monimiaceae & Mollinedia salicifolia Perkins & - & - & - & 5 & 0,48 & 0,96 \\
\hline Moraceae & Ficus gomeleira Kunth \& C.D.Bouché & 1 & 0,18 & 0,03 & 1 & 0,1 & 0,16 \\
\hline Moraceae & Helicostylis tomentosa (Poepp. \& Endl.) Rusby & 17 & 3,05 & 2,92 & 1 & 0,1 & 0,05 \\
\hline Moraceae & Pourouma guianensis Aubl. & - & - & - & 5 & 0,48 & 1,37 \\
\hline Moraceae & Sorocea bonplandii (Baill.) W.Burger & 1 & 0,18 & 0,1 & 3 & 0,29 & 0,15 \\
\hline Moraceae & Sorocea racemosa Gaudich. & - & - & - & 12 & 1,15 & 0,06 \\
\hline Myrsinaceae & Myrsinaceae sp01 & - & - & - & 1 & 0,1 & 0,03 \\
\hline Myrsinaceae & Cybianthus sp01 & 1 & 0,18 & 0,01 & - & - & - \\
\hline Myrsinaceae & Cybianthus amplus (Mez) G.Agostini & - & - & - & 2 & 0,19 & 0,06 \\
\hline Myrsinaceae & Cybianthus oblongifolius (A.DC.) G.Agostini & - & - & - & 7 & 0,67 & 0,08 \\
\hline Myrsinaceae & Myrsine sp01 & 1 & 0,18 & 0,02 & 1 & 0,1 & 0,03 \\
\hline Myrsinaceae & $\begin{array}{c}\text { Myrsine hermogenesii (Jung-Mend. \& Bernacci) } \\
\text { M.F.Freitas }\end{array}$ & - & - & - & 1 & 0,1 & 0,02 \\
\hline Myrsinaceae & Myrsine umbellata Mart. & - & - & - & 3 & 0,29 & 0,06 \\
\hline Myrtaceae & Myrtaceae sp01 & 1 & 0,18 & 0,04 & - & - & - \\
\hline Myrtaceae & Myrtaceae sp02 & 2 & 0,36 & 0,04 & 3 & 0,29 & 0,05 \\
\hline Myrtaceae & Myrtaceae sp03 & - & - & - & 2 & 0,19 & 0,06 \\
\hline Myrtaceae & Campomanesia dichotoma (O.Berg) Mattos & - & - & - & 1 & 0,1 & 0,02 \\
\hline
\end{tabular}


Tabela 1. Continuação.

\begin{tabular}{|c|c|c|c|c|c|c|c|}
\hline Família & Espécies & $\begin{array}{c}500 \mathrm{~m} \\
\mathrm{NI}\end{array}$ & $\begin{array}{c}500 \mathrm{~m} \\
\text { DeR }\end{array}$ & $\begin{array}{c}500 \mathrm{~m} \\
\text { DoR }\end{array}$ & $\begin{array}{c}800 \mathrm{~m} \\
\mathrm{NI}\end{array}$ & $\begin{array}{c}800 \mathrm{~m} \\
\text { DeR }\end{array}$ & $\begin{array}{c}800 \mathrm{~m} \\
\text { DoR }\end{array}$ \\
\hline Myrtaceae & Eugenia itapemirimensis Cambess. & 2 & 0,36 & 0,03 & 9 & 0,86 & 0,46 \\
\hline Myrtaceae & Eugenia sp01 & 3 & 0,54 & 0,05 & - & - & - \\
\hline Myrtaceae & Eugenia sp02 & 1 & 0,18 & 0 & - & - & - \\
\hline Myrtaceae & Eugenia sp03 & - & - & - & 1 & 0,1 & 0,17 \\
\hline Myrtaceae & Eugenia sp04 & 1 & 0,18 & 0,2 & 1 & 0,1 & 0,02 \\
\hline Myrtaceae & Eugenia sp05 & - & - & - & 6 & 0,57 & 0,7 \\
\hline Myrtaceae & Eugenia sp06 & 2 & 0,36 & 0,03 & - & - & - \\
\hline Myrtaceae & Eugenia sp07 & - & - & - & 2 & 0,19 & 0,01 \\
\hline Myrtaceae & Eugenia sp08 & - & - & - & 2 & 0,19 & 0,03 \\
\hline Myrtaceae & Eugenia sp09 & 12 & 2,15 & 0,8 & 1 & 0,1 & 0,04 \\
\hline Myrtaceae & Eugenia sp10 & 1 & 0,18 & 0,03 & - & - & - \\
\hline Myrtaceae & Eugenia sp11 & 1 & 0,18 & 0,02 & - & - & - \\
\hline Myrtaceae & Eugenia sp12 & 1 & 0,18 & 0,01 & - & - & - \\
\hline Myrtaceae & Eugenia oblongata O.Berg & - & - & - & 1 & 0,1 & 0 \\
\hline Myrtaceae & Eugenia schottiana O.Berg & - & - & - & 41 & 3,92 & 1,48 \\
\hline Myrtaceae & Eugenia tinguyensis Cambess. & 3 & 0,54 & 0,08 & 2 & 0,19 & 0,16 \\
\hline Myrtaceae & Marlierea sp01 & 2 & 0,36 & 0,9 & - & - & - \\
\hline Myrtaceae & Marlierea sp02 & 1 & 0,18 & 0,01 & - & - & - \\
\hline Myrtaceae & Marlierea sp03 & 1 & 0,18 & 0,01 & 2 & 0,19 & 0,06 \\
\hline Myrtaceae & Marlierea obversa D.Legrand & 5 & 0,9 & 0,15 & 4 & 0,38 & 0,23 \\
\hline Myrtaceae & Myrceugenia sp01 & - & - & - & 1 & 0,1 & 0 \\
\hline Myrtaceae & Myrceugenia miersiana (Gardner) D.Legrand \& Kausel & - & - & - & 2 & 0,19 & 0,04 \\
\hline Myrtaceae & Myrceugenia pilotantha (Kiaersk.) Landrum & - & - & - & 2 & 0,19 & 0,01 \\
\hline Myrtaceae & Myrcia sp01 & - & - & - & 1 & 0,1 & 0 \\
\hline Myrtaceae & Myrcia sp02 & - & - & - & 4 & 0,38 & 0,67 \\
\hline Myrtaceae & Myrcia racemosa (O.Berg) Kiaersk. & 1 & 0,18 & 0,02 & 3 & 0,29 & 0,13 \\
\hline Myrtaceae & Myrcia splendens (SW.) DC. & - & - & - & 2 & 0,19 & 0,01 \\
\hline Myrtaceae & Myrciaria floribunda (H.West ex Willd.) O.Berg & 4 & 0,72 & 0,83 & 3 & 0,29 & 3,58 \\
\hline Myrtaceae & Plinia sp01 & - & - & - & 2 & 0,19 & 0,03 \\
\hline Myrtaceae & Plinia rara Sobral & - & - & - & 6 & 0,57 & 0,04 \\
\hline Myrtaceae & Plinia rivularis (Cambess.) Rotman & 4 & 0,72 & 0,12 & - & - & - \\
\hline Nyctaginaceae & Guapira laxiflora (Choisy) Lundell & 9 & 1,61 & 0,13 & 8 & 0,76 & 0,6 \\
\hline Nyctaginaceae & Guapira obtusata (Jacq.) Little & 6 & 1,08 & 0,1 & 41 & 3,92 & 9,77 \\
\hline Nyctaginaceae & Guapira opposita (Vell.) Reitz & 21 & 3,76 & 0,42 & 17 & 1,63 & 1,82 \\
\hline Ochnaceae & Ouratea gigantophylla (Erhard) Engl. & - & - & - & 2 & 0,19 & 0,15 \\
\hline Olacaceae & Olacaceae sp01 & 1 & 0,18 & 0,77 & - & - & - \\
\hline Olacaceae & Olacaceae sp02 & 2 & 0,36 & 0,01 & - & - & - \\
\hline Olacaceae & Heisteria sp01 & 2 & 0,36 & 0,02 & - & - & - \\
\hline Olacaceae & Heisteria silvianii Schwacke & - & - & - & 1 & 0,1 & 0,09 \\
\hline Olacaceae & Tetrastylidium grandifolium (Baill.) Sleumer & 8 & 1,43 & 1,98 & 4 & 0,38 & 0,39 \\
\hline Oleaceae & Chionanthus micranthus (Eichler) Lozano \& Fuertes & 1 & 0,18 & 0,02 & 1 & 0,1 & 0,02 \\
\hline Passifloraceae & Passiflora contracta Vitta & - & - & - & 1 & 0,1 & 0,03 \\
\hline Phyllanthaceae & Hieronyma alchorneoides M.Allemão & 1 & 0,18 & 0,02 & 1 & 0,1 & 0,01 \\
\hline Phyllanthaceae & Hieronyma oblonga Müll. Arg. & - & - & - & 5 & 0,48 & 3,54 \\
\hline
\end{tabular}


Tabela 1. Continuação.

\begin{tabular}{|c|c|c|c|c|c|c|c|}
\hline Família & Espécies & $\begin{array}{c}500 \mathrm{~m} \\
\mathrm{NI}\end{array}$ & $\begin{array}{c}500 \mathrm{~m} \\
\text { DeR }\end{array}$ & $\begin{array}{c}500 \mathrm{~m} \\
\text { DoR }\end{array}$ & $\begin{array}{c}800 \mathrm{~m} \\
\text { NI }\end{array}$ & $\begin{array}{c}800 \mathrm{~m} \\
\mathrm{DeR}\end{array}$ & $\begin{array}{c}800 \mathrm{~m} \\
\text { DoR }\end{array}$ \\
\hline Phyllanthaceae & Margaritaria nobilis L.f. & - & - & - & 1 & 0,1 & 0,07 \\
\hline Picramniaceae & Picramnia glazioviana Engl. & 1 & 0,18 & 0,05 & - & - & - \\
\hline Piperaceae & Piper bowiei Yuncker & - & - & - & 1 & 0,1 & 0,01 \\
\hline Piperaceae & Piper richardiifolium Kunth & - & - & - & 16 & 1,53 & 0,43 \\
\hline Poaceae & Chusquea bambusoides (Raddi) Hack. & 17 & 3,05 & 0,96 & - & - & - \\
\hline Polygonaceae & Coccoloba sp01 & - & - & - & 1 & 0,1 & 0 \\
\hline Polygonaceae & Coccoloba mosenii Lind. & - & - & - & 5 & 0,48 & 0,27 \\
\hline Proteaceae & Euplassa sp01 & - & - & - & 1 & 0,1 & 0,11 \\
\hline Quiinaceae & Lacunaria decastyla Ducke & 2 & 0,36 & 0 & - & - & - \\
\hline Quiinaceae & Quiina magallano-gomesii Schwacke & - & - & - & 2 & 0,19 & 0,01 \\
\hline Rosaceae & Prunus myrtifolia (L.) Urban & 1 & 0,18 & 0,04 & 3 & 0,29 & 0,99 \\
\hline Rubiaceae & Rubiaceae sp01 & 1 & 0,18 & 0,01 & - & - & - \\
\hline Rubiaceae & Amaioua guianensis Aubl. & - & - & - & 10 & 0,96 & 0,47 \\
\hline Rubiaceae & Amaioua intermedia Mart. & 2 & 0,36 & 0,14 & - & - & - \\
\hline Rubiaceae & Bathysa cuspidata (A.St.-Hil.) Hook.f. ex K.Schum. & 1 & 0,18 & 0 & 13 & 1,24 & 0,38 \\
\hline Rubiaceae & Bathysa mendoncaei K.Schum. & 16 & 2,87 & 0,33 & 36 & 3,44 & 1,31 \\
\hline Rubiaceae & Chomelia pubescens Cham. \& Schltdl. & - & - & - & 6 & 0,57 & 0,08 \\
\hline Rubiaceae & Cordieria sp01 & 1 & 0,18 & 0 & - & - & - \\
\hline Rubiaceae & Coussarea contracta Müll.Arg. & - & - & - & 2 & 0,19 & 0,02 \\
\hline Rubiaceae & Coussarea ilheotica Müll.Arg. & - & - & - & 11 & 1,05 & 0,41 \\
\hline Rubiaceae & Faramea bahiensis Müll.Arg. & 2 & 0,36 & 0,01 & - & - & - \\
\hline Rubiaceae & Faramea martiana Müll.Arg. & - & - & - & 11 & 1,05 & 0,09 \\
\hline Rubiaceae & Faramea sp01 & 5 & 0,9 & 0,22 & - & - & - \\
\hline Rubiaceae & Ferdinandusa edmundoi Sucre & 3 & 0,54 & 0,08 & - & - & - \\
\hline Rubiaceae & Ixora sp01 & 7 & 1,25 & 1,83 & - & - & - \\
\hline Rubiaceae & Malanea sp01 & - & - & - & 1 & 0,1 & 0 \\
\hline Rubiaceae & Margaritopsis chaenotricha (DC.) C.M.Taylor & - & - & - & 2 & 0,19 & 0,01 \\
\hline Rubiaceae & Palicourea blanchetiana Schltdl. & - & - & - & 1 & 0,1 & 0 \\
\hline Rubiaceae & Posoqueria acutifolia Mart. & - & - & - & 2 & 0,19 & 0,46 \\
\hline Rubiaceae & Psychotria carthagenensis Jacq. & - & - & - & 9 & 0,86 & 0,09 \\
\hline Rubiaceae & Psychotria colorata (Willd. ex Roem. \& Schult.) Müll.Arg. & - & - & - & 1 & 0,1 & 0,01 \\
\hline Rubiaceae & Psychotria leiocarpa Cham. \& Schltdl. & 1 & 0,18 & 0 & 2 & 0,19 & 0,01 \\
\hline Rubiaceae & Psychotria mapourioides DC. & 1 & 0,18 & 0,01 & 3 & 0,29 & 0,16 \\
\hline Rubiaceae & Psychotria myriantha Müll.Arg. & 1 & 0,18 & 0,01 & 4 & 0,38 & 0,42 \\
\hline Rubiaceae & Psychotria nemorosa Gardner & - & - & - & 2 & 0,19 & 0,02 \\
\hline Rubiaceae & Psychotria pleiocephala Müll.Arg. & - & - & - & 11 & 1,05 & 1,09 \\
\hline Rubiaceae & Psychotria suterella Müll.Arg. & - & - & - & 8 & 0,76 & 0,09 \\
\hline Rubiaceae & Randia sp01 & 2 & 0,32 & 0,04 & - & - & - \\
\hline Rubiaceae & Randia armata (Sw.) DC. & - & - & - & 8 & 0,76 & 0,06 \\
\hline Rubiaceae & Rudgea sp01 & - & - & - & 2 & 0,19 & 0,01 \\
\hline Rubiaceae & Rudgea sp02 & 4 & 0,72 & 0,04 & 4 & 0,38 & 0,03 \\
\hline Rubiaceae & Rudgea celastrinea Müll.Arg. & 6 & 1,08 & 0,25 & - & - & - \\
\hline Rubiaceae & Rudgea reticulata Benth. & 5 & 0,9 & 0,36 & - & - & - \\
\hline Rubiaceae & Schradera polycephala DC. & - & - & - & 1 & 0,1 & 0 \\
\hline Rutaceae & Dictyoloma vandellianum A.Juss. & - & - & - & 1 & 0,1 & 0,21 \\
\hline Sabiaceae & Meliosma sp01 & - & - & - & 1 & 0,1 & 0 \\
\hline Salicaceae & Banara serrata (Vell.) Warb. & - & - & - & 1 & 0,1 & 0 \\
\hline Salicaceae & Casearia commersoniana Cambess. & 5 & 0,9 & 0,8 & - & - & - \\
\hline
\end{tabular}


Tabela 1. Continuação.

\begin{tabular}{|c|c|c|c|c|c|c|c|}
\hline Família & Espécies & $\begin{array}{c}500 \mathrm{~m} \\
\mathrm{NI}\end{array}$ & $\begin{array}{c}500 \mathrm{~m} \\
\text { DeR }\end{array}$ & $\begin{array}{c}500 \mathrm{~m} \\
\text { DoR }\end{array}$ & $\begin{array}{c}800 \mathrm{~m} \\
\mathrm{NI}\end{array}$ & $\begin{array}{c}800 \mathrm{~m} \\
\text { DeR }\end{array}$ & $\begin{array}{c}800 \mathrm{~m} \\
\text { DoR }\end{array}$ \\
\hline Salicaceae & Casearia decandra Jacq. & - & - & - & 2 & 0,19 & 0,52 \\
\hline Salicaceae & Casearia oblongifolia Cambess. & 2 & 0,36 & 0,02 & - & - & - \\
\hline Sapindaceae & Sapindaceae sp01 & - & - & - & 1 & 0,1 & 0,01 \\
\hline Sapindaceae & Sapindaceae sp02 & - & - & - & 2 & 0,19 & 0,02 \\
\hline Sapindaceae & Allophylus petiolulatus Radlk. & - & - & - & 4 & 0,38 & 1,49 \\
\hline Sapindaceae & Cupania furfuracea Radlk. & 2 & 0,36 & 4,69 & 13 & 1,24 & 6,22 \\
\hline Sapindaceae & Cupania rugosa Radlk. & 1 & 0,18 & 1,62 & 2 & 0,19 & 0,04 \\
\hline Sapindaceae & Matayba guianensis Aubl. & 1 & 0,18 & 0,01 & 9 & 0,86 & 0,34 \\
\hline Sapindaceae & Paullinia carpopoda Cambess. & - & - & - & 6 & 0,57 & 0,23 \\
\hline Sapindaceae & Paullinia micrantha Cambess. & - & - & - & 1 & 0,1 & 0 \\
\hline Sapotaceae & Sapotaceae sp01 & 2 & 0,36 & 0,75 & - & - & - \\
\hline Sapotaceae & Sapotaceae sp02 & 3 & 0,54 & 0,16 & - & - & - \\
\hline Sapotaceae & Sapotaceae sp03 & 1 & 0,18 & 0,47 & - & - & - \\
\hline Sapotaceae & Sapotaceae sp04 & 1 & 0,18 & 0,02 & - & - & - \\
\hline Sapotaceae & Sapotaceae sp05 & 1 & 0,18 & 0,15 & - & - & - \\
\hline Sapotaceae & Sapotaceae sp06 & - & - & - & 1 & 0,1 & 0,01 \\
\hline Sapotaceae & Chrysophyllum splendens Spreng. & 2 & 0,36 & 0,06 & - & - & - \\
\hline Sapotaceae & Diploon cuspidatum (Hoehne) Cronq. & 2 & 0,36 & 2,01 & 8 & 0,76 & 3,06 \\
\hline Sapotaceae & Ecclinusa ramiflora Mart. & 5 & 0,9 & 1,1 & 22 & 2,1 & 1,79 \\
\hline Sapotaceae & Micropholis compta Pierre & 1 & 0,18 & 0,04 & - & - & - \\
\hline Sapotaceae & Pouteria sp01 & 1 & 0,18 & 0,06 & - & - & - \\
\hline Sapotaceae & Pouteria sp02 & 1 & 0,18 & 0,09 & - & - & - \\
\hline Sapotaceae & Pouteria bangii (Rusby) T.D.Penn. & 3 & 0,54 & 1,52 & - & - & - \\
\hline Sapotaceae & Pouteria beaurepairei (Glaz. \& Raunk.) Baehni & 1 & 0,18 & 0,08 & 1 & 0,1 & 0,01 \\
\hline Sapotaceae & Pouteria butyrocarpa (Kuhlm.) T.D.Penn. & - & - & - & 1 & 0,1 & 0,01 \\
\hline Sapotaceae & Pouteria grandiflora (A.DC.) Baehni & 3 & 0,54 & 0,02 & - & - & - \\
\hline Sapotaceae & Pouteria torta (Mart.) Radlk. & 2 & 0,36 & 0,13 & 1 & 0,1 & 0,04 \\
\hline Sapotaceae & Pradosia lactescens (Vell.) Radlk. & 5 & 0,9 & 0,46 & - & - & - \\
\hline Schlegeliaceae & Schlegelia parviflora (Derst.) Monachino & - & - & - & 2 & 0,19 & 0,12 \\
\hline Simaroubaceae & Simaba guianensis Aubl. & 2 & 0,36 & 0,69 & - & - & - \\
\hline Solanaceae & Aureliana fasciculata (Vell.) Sendtn. & - & - & - & 4 & 0,38 & 0,02 \\
\hline Solanaceae & Cestrum salzmannii Dunal. & - & - & - & 7 & 0,67 & 0,32 \\
\hline Solanaceae & Solanaceae sp01 & 1 & 0,18 & 0,2 & - & - & - \\
\hline Solanaceae & Solanum rupincola Sendtn. & - & - & - & 1 & 0,1 & 0 \\
\hline Solanaceae & Solanum swartzianum Roem. \& Schult. & - & - & - & 3 & 0,29 & 0,1 \\
\hline Styracaceae & Styrax sp01 & - & - & - & 1 & 0,1 & 0,03 \\
\hline Symplocaceae & Symplocos estrellensis Casar. & 1 & 0,18 & 0,01 & 7 & 0,67 & 0,27 \\
\hline Theaceae & Laplacea fruticosa (Schrad.) Kobuski & - & - & - & 2 & 0,19 & 0,43 \\
\hline Urticaceae & Cecropia glaziovii Snethl. & 11 & 1,97 & 1,64 & 12 & 1,15 & 0,64 \\
\hline Urticaceae & Cecropia hololeuca Miq. & 4 & 0,72 & 0,25 & 1 & 0,1 & 0,31 \\
\hline Violaceae & Rinorea guianensis Aubl. & 13 & 2,33 & 0,97 & - & - & - \\
\hline Vitaceae & Cissus sp01 & 1 & 0,18 & 0 & - & - & - \\
\hline Vochysiaceae & Qualea sp01 & 2 & 0,36 & 0,06 & - & - & - \\
\hline Vochysiaceae & Vochysia rectiflora Warm. & 1 & 0,18 & 1,4 & - & - & - \\
\hline Vochysiaceae & Vochysia riedeliana Stafleu & 10 & 1,79 & 11,58 & - & - & - \\
\hline
\end{tabular}


caram ainda, uma elevada riqueza em Melastomataceae e o decréscimo de espécies em Sapotaceae em áreas de altitude, corroborando com os dados aqui obtidos.

Com relação à riqueza específica, a área 1 apresentou maior número de espécies do que a área 2 (Tab. 2). Isso, no entanto, se relaciona com o tamanho da amostra, pois quando se padroniza o esforço, através das curvas de rarefação (Fig. 4), percebe-se que a área 2 pode ser considerada como a mais rica (Fig. 4A). A redução no número de espécies com o aumento da altitude é um padrão comumente encontrado em estudos de estrutura arbórea em floresta tropical (Lieberman et al. 1996; Moreno et al. 2003; Meireles et al. 2008). Alguns autores (Lieberman et al. 1996; Pendry \& Proctor 1996) atribuem este fato às condições ambientais se tornarem mais rigorosas com incremento da altitude (i.e. fatores edáficos, temperatura, velocidade dos ventos e precipitação) e isto pode ser percebido na RPPN Serra Bonita, ainda que empiricamente.

As alturas médias não variaram significativamente entre as duas áreas, pois apresentaram valor similar com alto desvio padrão. Entretanto a análise da estrutura vertical evidencia a diferença na distribuição do número de indivíduos nas classes de altura entre as diferentes altitudes (Fig. 5). Há uma predominância das classes $<5 \mathrm{~m}$ e de $5+10 \mathrm{~m}$ na área 1 e, em contraposição, há uma menor abundância na classe inicial de altura na área 2. Além disso, nesta área existe uma maior representatividade da classe $\geq 30 \mathrm{~m}$, evidenciando modificações nos principais estratos da floresta entre os dois trechos. Em seu sistema de classificação da vegetação brasileira Veloso et al. (1991) mencionam que em floresta Submontana a presença de nanofanerófitos é pequena e sua principal característica são os fanerófitos de grande porte, enquanto que na floresta Montana, esta forma de vida tende a apresentar um porte menor. Assim, o padrão de estrutura detectado, indica que as delimitações propostas por estes autores podem ser percebidas na área estudada.

A verificação de uma maior densidade de indivíduos na área 1 (3493 ind./ha) pode ser atribuída à alta concentração destes nas classes de altura iniciais. Entretanto, a área basal por hectare foi maior na área $2\left(71,54 \mathrm{~cm}^{2} / \mathrm{ha}\right)$. A relação inversa entre densidade e área basal é consequência da existência de árvores com maior diâmetro e menos indivíduos no sub-bosque na área locada em menor altitude. Em contraposição, na área 1 o sub-bosque mostra-se mais populoso, o que nos permite inferir que, com o aumento da altitude, a tendência da floresta é apresentar maior número de indivíduos e estes apresentarem menor diâmetro. Essa variação recebe influência direta de Rubiaceae e Myrtaceae que, na área 1 possuem maior riqueza e abundância de indivíduos com menos de $5 \mathrm{~m}$ de altura. Por outro lado, na área 2 esta classe de altura possui, além de muitas espécies de Rubiaceae e Fabaceae, indivíduos jovens de Guapira opposita (Nyctaginaceae) que dominaram em abundância.

A composição florística entre as duas áreas também pode ser considerada distinta visto que, na análise de agrupamen- to, os cinco plots se separam em dois grupos, sustentados por um alto valor de bootstrap (Fig. 6). As diferenças em riqueza, densidade de indivíduos e estrutura apontada anteriormente entre essas áreas, sugerem que a RPPN Serra Bonita é formada por um mosaico de comunidades distintas, mas pertencentes a uma mesma fitofisionomia. E dentro de cada área, há manchas que se pode considerar como uma composição peculiar como evidenciado na área 1 , onde mesmo os plots $\mathrm{T} 1$ e T2, alocados em áreas próximas possuem uma similaridade menor do que os plots T1 e T3 que se posicionam mais isoladamente.

Apesar de serem verificadas diferenças na vegetação ocorrida entre as altitudes, o mesmo não aconteceu com os parâmetros medidos no solo. A composição química (Tab. 3) foi similar nas duas áreas onde os valores de $\mathrm{pH}$, $\mathrm{P}$ e $\mathrm{Mg}$ não apresentaram variações evidentes. $\mathrm{O} \mathrm{Ca}$ e a composição física (Tab. 4) apresentaram sutis alterações, não chegando, entretanto, a caracterizar outra classe de solo. Desse modo consideramos que existem outros fatores, que não os edáficos, influenciando na estrutura e composição da vegetação na RPPN Serra Bonita. É possível que os fatores mais influentes sobre estas diferenças no estabelecimento das espécies sejam realmente a temperatura e precipitação, que de acordo com Gotelli et al. (2009) são as variáveis mais correlacionadas com os padrões de distribuição da riqueza de espécies em biomas terrestres.

A distância, em linha reta, de aproximadamente $800 \mathrm{~m}$ entre a área 1 e a 2, aliado ao baixo compartilhamento de espécies (i.e. 71 espécies perfazendo ca. 20\%) sugere que a composição florística sofre uma mudança abrupta. Esta mudança pode estar influenciada não só pela altitude, mas também por uma topografia local acidentada, gerando diferentes micro-habitats que poderia apresentar fatores que possam inibir o estabelecimento de determinadas espécies. Desta forma, populações que agregam espécies altamente abundantes, ficam mais restritas a determinados ambientes a exemplo de Cyathea phalerata, Eugenia schottiana, Geonoma gamiova e Piper richardifolium que ocorreram apenas na área 1 ao contrário de Cyathea corcovadensis, Eriotheca macrophylla, Rinorea guianensis e Vochysia riedeliana, que foram encontradas somente na área 2. Em contraposição, espécies comumente detectadas em florestas úmidas no sul da Bahia como Bathysa cuspidata e Euterpe edulis, foram encontradas com elevada abundância nas duas áreas (Martini et al. 2007; Thomas et al. 2009).

Informações florísticas e de estrutura envolvendo outras formas de vida são escassas na RPPN Serra Bonita. Nesse ambiente, Amorim et al. (2009) documentaram mais de 132 espécies de angiospermas epífitas, uma delas publicada como nova por Amorim \& Leme (2009). Matos (2009) ressalta elevada riqueza de samambaias epífitas nesta área, indicando 22 registros inéditos no nordeste do Brasil (Matos et al. 2010a) e a ocorrência de uma nova espécie (Matos et al. 2010b). Em trabalho precursor sobre estrutura filogenética e redes de interação envolvendo 
Tabela 2. Parâmetros gerais das duas áreas amostradas na RPPN Serra Bonita, Bahia, Brasil.

\begin{tabular}{|c|c|c|c|}
\hline Parâmetros & Total & Área 1 & Área 2 \\
\hline Área total (ha) & 0,5 & 0,3 & 0,2 \\
\hline Número de indivíduos & 1604 & 1046 & 558 \\
\hline Número de espécies & 355 & 224 & 204 \\
\hline Número de famílias & 68 & 55 & 55 \\
\hline Índice Shannon (H') & 5,261 & 4,818 & 4,897 \\
\hline Equabilidade $\left(J=H^{\prime} / \ln (S)\right)$ & 0,896 & 0,890 & 0,921 \\
\hline Densidade (ind./ha) & 3208 & 3487 & 2790 \\
\hline Área basal (m²/ha) & 59,78 & 51,89 & 71,63 \\
\hline Diâmetro médio $(\mathrm{cm}) \pm$ d.p. & $10,04 \pm 11,9$ & $9,28 \pm 10,17$ & $11,45 \pm 14,00$ \\
\hline Altura média $(\mathrm{m}) \pm$ d.p. & $8,33 \pm 6,28$ & $7,97 \pm 6,11$ & $8,99 \pm 6,53$ \\
\hline
\end{tabular}

samambaias, Mortara (2011) detectaram aspectos das relações de co-ocorrência entre e as formas de vida e, no caso das epífitas, as complexas relações inter e intra-metacomunidades. Assim, devido à alta frequência de epífitas em árvores de grande porte, associados à ausência de vestígios de extração madeireira e ao alto valor de área basal obtido (> $50 \mathrm{~m}^{2} / \mathrm{ha}$ ), considera-se que os trechos de floresta onde os plots foram alocadas podem ser indicados como maduros de acordo com critérios apontados por Clark (1996).

Outra forma de vida investigada, as lianas (i.e. trepadeiras lenhosas), foram apontadas como uma das principais diferenças entre as florestas tropicais e temperadas (Croat 1978 apud Lima et al. 1997). Este fato reforça a importância da inclusão dessas formas de vida em amostragens de estrutura. Mesmo assim, a maior parte dos estudos existentes em áreas tropicais negligencia este grupo. O critério de inclusão possibilitou apenas o registro das lianas, que de acordo com Lima et al. (1997) são abundantes em áreas primárias.

Levando-se em consideração somente a composição de espécies de lianas, verifica-se que apenas três espécies (das 44 existentes) são compartilhadas, duas pertencentes à Fabaceae (Machaerium uncinatum e Fabaceae sp01) e Mikania mattos-silvae (Asteraceae). Com a análise das curvas de rarefação percebe-se uma diferença na riqueza de lianas entre essas áreas. O número de espécies da área 2 está abaixo do intervalo de confiança do esperado para a área 1 com o mesmo número de indivíduos sendo o oposto do observado para a comunidade como um todo (Fig. 4B). A maior riqueza de lianas na área 1 pode estar relacionada com a quantidade de indivíduos arbustivo/arbóreo de pequeno porte que servem de apoio para os indivíduos mais jovens de lianas, pois segundo Nabe-Nielsen (2001) as áreas com alta densidade de árvores de pequeno porte possuem alta densidade e diversidade de lianas. Apesar das lianas serem apontadas como indicadores de perturbação antrópica, no presente trabalho foram encontrados indivíduos com DAP $>10 \mathrm{~cm}$.
Além das lianas, outra forma de crescimento que merece atenção em áreas tropicais são os bambus lenhosos. Espécies de Poaceae (Bambusoideae) são consideradas invasoras de áreas perturbadas (Silveira 2001), alterando a dinâmica e a estrutura das populações e comunidades (Veblen 1982; Kiyoshi et al. 1996; Silveira 2001). Isto poderia induzir, equivocadamente, a ideia de que a área 2, por apresentar em alguns trechos alta abundância de Chusquea bambusoides, seria uma floresta com amplo grau de antropização. Contudo, Young (1991) concluiu que este gênero não é típico de clareiras, devido à sua intolerância ao sol. Mota et al. (2009) confirmam que esta espécie na RPPN Serra Bonita é nativa e comum no interior de floresta, onde percebe-se a sua ausência em áreas degradadas. Desta forma, a elevada abundância de C. bambusoides deve ser considerada como natural.

Analisando os perfis de diversidade percebe-se que as curvas não se cruzam em nenhuma das situações (Fig. 7 A, B e C). Estes perfís obtidos mostram que as duas comunidades podem ser ranqueadas em termos de diversidade, sendo consideradas comparáveis por Tóthmérész (1995) ou separáveis (Liu et al. 2007). Desta forma, é possível inferir que a área 2 possui maior equabilidade e riqueza do que a área 1. Isto é conseqüência da existência de três espécies predominantes (Cyathea phalerata, Eugenia schottiana e Guapira obtusata) e de uma menor abundância de espécies raras na área 1. Esta análise seguiu a indicação de autores que sugerem a utilização deste método de ordenação da diversidade, pois a utilização de um único índice poderia trazer conclusões incorretas a este respeito (Lewis et al. 1988; Kindt et al. 2006; Melo 2008).

Quando comparada a riqueza total obtida na RPPN Serra Bonita com alguns trabalhos recentes em florestas úmidas (Fig. 8), padronizando o critério de inclusão (DAP $>5 \mathrm{~cm}$ ) e o esforço amostral (curva de rarefação), os resultados indicam maior proximidade com os existentes no sul da Bahia (e.g. Martini et al. 2007; Thomas et al. 2009) do que com os do sudeste [e.g. Moreno et al. (2003) na Serra do Imbé, RJ 
Tabela 3. Resultado das análises químicas do solo do trecho estudado nas duas áreas amostradas na RPPN Serra Bonita, Bahia, Brasil (T1 - plot 1, T2 - plot 2,...).

\begin{tabular}{cllllllll}
\hline Plot & Profund. & $\mathrm{pH}$ & $\mathrm{Al}$ & $\mathrm{Ca}$ & $\mathrm{Mg}$ & $\mathrm{K}$ & $\mathrm{C}$ & $\mathrm{P}$ \\
\hline & $\mathrm{cm}$ & & & & $\mathrm{cmol} / \mathrm{dm}^{3}$ & & \\
T1 & $0-20$ & 4,4 & 1,7 & 0,5 & 0,4 & 0,17 & 36,72 & 2 \\
T2 & $0-20$ & 4,2 & 2,4 & 0,4 & 0,4 & 0,12 & 35,76 & 2 \\
T3 & $0-20$ & 4,3 & 1,7 & 0,1 & 0,1 & 0,04 & 27,60 & 1 \\
T4 & $0-20$ & 4,1 & 1,9 & 0,5 & 0,3 & 0,13 & 27,36 & 2 \\
T5 & $0-20$ & 4,4 & 1,1 & 0,9 & 0,6 & 0,13 & 32,76 & 2 \\
T1 & $20-40$ & 4,3 & 1,0 & 0,1 & 0,1 & 0,07 & 22,08 & 1 \\
T2 & $20-40$ & 4,3 & 1,4 & 0,1 & 0,1 & 0,06 & 32,16 & 1 \\
T3 & $20-40$ & 4,4 & 1,2 & 0,0 & 0,0 & 0,02 & & 17,64 \\
T4 & $20-40$ & 4,1 & 1,7 & 0,3 & 0,2 & 0,09 & 24,72 & 1 \\
T5 & $20-40$ & 4,3 & 1,1 & 0,2 & 0,2 & 0,08 & 18,84 & 2 \\
\hline
\end{tabular}

Tabela 4. Resultado das análises físicas do solo do trecho estudado nas duas áreas amostradas na RPPN Serra Bonita, Bahia, Brasil. (T1 - plot 1, T2 - plot 2,...).

\begin{tabular}{|c|c|c|c|c|c|c|c|c|c|}
\hline Plot. & Profund. & Areia Grossa & $\begin{array}{c}\text { Areia } \\
\text { Fina }\end{array}$ & Silte & Argila & Silte/Argila & Grau Flocul. & Umid. Equiv. & Dens. real \\
\hline & $\mathrm{cm}$ & & & $\mathrm{g} / \mathrm{kg}$ & & & $\%$ & $\mathrm{~g} / \mathrm{kg}$ & $\mathrm{g} / \mathrm{cm}^{3}$ \\
\hline $\mathrm{T} 1$ & $0-20$ & 510 & 145 & 80 & 265 & 0,30 & 86,8 & 220,9 & 2,58 \\
\hline $\mathrm{T} 2$ & $0-20$ & 389 & 133 & 280 & 198 & 1,41 & 90,4 & 219,5 & 2,63 \\
\hline $\mathrm{T} 3$ & $0-20$ & 525 & 114 & 210 & 151 & 1,39 & 79,5 & 183,6 & 2,60 \\
\hline $\mathrm{T} 4$ & $0-20$ & 329 & 72 & 229 & 370 & 0,62 & 90,0 & 252,1 & 2,60 \\
\hline T5 & $0-20$ & 335 & 78 & 275 & 312 & 0,88 & 89,7 & 276,1 & 2,69 \\
\hline $\mathrm{T} 1$ & $20-40$ & 347 & 125 & 345 & 183 & 1,88 & 86,3 & 218,0 & 2,64 \\
\hline $\mathrm{T} 2$ & $20-40$ & 414 & 121 & 261 & 204 & 1,28 & 94,1 & 176,7 & 2,62 \\
\hline $\mathrm{T} 3$ & $20-40$ & 437 & 103 & 196 & 264 & 0,74 & 58,0 & 215,4 & 2,67 \\
\hline $\mathrm{T} 4$ & $20-40$ & 268 & 82 & 289 & 361 & 0,80 & 87,5 & 258,8 & 2,67 \\
\hline T5 & $20-40$ & 299 & 75 & 265 & 361 & 0,73 & 88,2 & 266,9 & 2,69 \\
\hline
\end{tabular}

e Meireles et al. (2008) na Serra da Mantiqueira, MG] além de um estudo no nordeste setentrional, a saber Serra da Borborema, PE (Ferraz \& Rodal 2006). Confirma-se assim, um elevado índice de riqueza na área de estudo ainda que este, permaneça em posição intermediária aos resultados obtidos em Martini et al. (2007) e Thomas et al. (2009) no Parque Estadual da Serra do Conduru e na RPPN Serra do Teimoso respectivamente, ambos na Bahia.

Comparações na composição florística em diferentes áreas de floresta no sul da Bahia ainda não foram executadas de modo criterioso. Verifica-se, entretanto, que a maior parte das espécies mais abundantes na RPPN Serra Bonita não ocorrem na região estudada por Thomas et al. (2009). Percebe-se também que as que ocorrem, possuem abundâncias completamente diferentes (i.e. quando maior abundância em uma área, menor na outra). Por outro lado, quando comparados com o estudo de Martini et al. (2007) e de Thomas et al. (2008), ambos realizados no Parque Estadual Serra do Conduru, verifica-se que algumas espécies (e.g. Ecclinusa ramiflora, Eriotheca macrophyla, Euterpe edulis e Helicostylis tomentosa) ocorrem em abundância na RPPN Serra Bonita. Isto pode ser reflexo do mosaico de manchas de habitats defendido por Dunning et al. (1992) ao elaborar um panorama sobre processos que afetam populações em paisagens complexas.

Desta forma, numa análise local, a RPPN Serra Bonita apresentou maior número de espécies em comum com o Parque Estadual Serra do Conduru (a ca. $150 \mathrm{~km}$ ) do que com a RPPN Serra do Teimoso (a ca. $25 \mathrm{~km}$ ). Com isso pode-se inferir que a proximidade geográfica nem sempre é um fator preponderante na semelhança entre a composição e a estrutura em comunidades arbóreas. Neste caso há uma suposição da influencia da umidade que vem do litoral, pois entre a RPPN Serra do Teimoso e o litoral há um complexo de montanhas (i.e. formações conhecidas como Serra das Lontras), enquanto a RPPN Serra Bonita é a primeira grande formação montanhosa a partir do mar para o interior do continente. Além disso, outro fator que influencia a composição da RPPN Serra do Teimoso é a presença de elementos de floresta estacional semidecidual nas áreas mais baixas com reflexo direto nas áreas úmidas mais altas (Thomas et al. 2009). Enquanto isso, na formação montanhosa da Serra Bonita a predominância é de floresta ombrófila, o mesmo acontecendo na área da Serra do Conduru. 
Boa parte da literatura existente para estudos de estrutura da comunidade em remanescentes de Floresta Atlântica utiliza DAP $\geq 5 \mathrm{~cm}$. Desse modo, com a investigação de alguns parâmetros baseados nesse critério de inclusão percebem-se alterações, algumas aqui apresentadas e discutidas. Com esse critério o número total de indivíduos cai para 920, sendo 537 na área 1 e 347 na área 2. Por outro lado a densidade se mantém elevada, especialmente quando comparada ao inventário de Thomas et al. (2009). As Melastomataceae sofrem uma sutil redução em sua riqueza, entretanto essa ainda é alta quando comparada aos estudos de Martini et al. (2007) e Thomas et al. (2009). Em Rubiaceae o total de espécies é reduzido a quase metade em ambas as áreas e isso pode ser explicado pela predominância natural de espécies com diâmetros reduzidos neste grupo. Considera-se então, que para ter idéia da estrutura da comunidade lenhosa de uma forma geral, a amostragem com critério de inclusão mais amplo pode ser adotada, pois assim é possível aumentar o número de indivíduos na amostra incluindo consequentemente um maior número de espécies.

$\mathrm{Na}$ Serra Bonita existem diferenças na vegetação dos dois trechos amostrados que são importantes na manutenção da diversidade encontrada na Floresta Atlântica, pois cada altitude retêm espécies exclusivas contribuindo assim para a elevada riqueza nas regiões montanhosas. Os resultados encontrados no presente estudo reforçam a recomendação de Martinelli (2007) sobre a necessidade de esforços para conservação das formações montanhosas especialmente no leste setentrional brasileiro, levando em consideração as diferenças entre as comunidades ao longo do gradiente de altitude em regiões como as ainda existentes no sul da Bahia. A RPPN Serra Bonita é importante remanescente para a preservação da biodiversidade das montanhas no Brasil servindo de exemplo para a criação de novas unidades de conservação, com foco nas regiões de altitude, além da ampliação das já existentes.

A baixa similaridade entre os plots amostrados (resultado do baixo compartilhamento de espécies), a diferença na riqueza, na densidade (ind./ha), na área basal $\left(\mathrm{m}^{2} / \mathrm{ha}\right.$ ) e na concentração de indivíduos nas classes de altura, nos leva a conclusão de que os dois trechos de florestas estudados são muito distintos. Contudo, se assemelham pelo bom grau de preservação, que é indicado pela alta abundância e riqueza de lianas, presença de espécies de valor comercial com grande porte (e.g. Arapatiella psilophylla, Caryocar edule, Copaifera trapaezifolia, Handroanthus heptaphyllus e Lecythis pisonis) e baixa abundância de espécies pioneiras. A alta diversidade também é característica importante compartilhada pelos dois trechos dessa floresta. Assim, contatou-se que as diferenças estruturais ocorrem de forma natural, porém ainda não estão claros os limites que levam a reconhecer sua heterogeneidade.

\section{Agradecimentos}

Ao Programa de Pós-Graduação em Botânica da Universidade Estadual de Feira de Santana pelo auxílio financeiro nas coletas; à Fundação de Amparo à Pesquisa do Estado da Bahia (FAPESB) pela bolsa de Mestrado concedida ao primeiro autor e pelo financiamento obtido no Edital de Pesquisas 2009; ao Conselho Nacional de Desenvolvimento Científico e Tecnológico (CNPq) pela bolsa de Produtividade concedida ao segundo autor e pelo financiamento obtido no Edital Universal 2009. Ao Sr. Vitor Becker e Clemira Souza, proprietários da RPPN Serra Bonita, pelo auxílio logístico; aos técnicos José Lima e Lukas Daneu pelo apoio durante o trabalho de campo. Assumimos a responsabilidade por todos os nomes de espécies apresentadas, mas somos gratos aos botânicos que identificaram e/ ou confirmaram parte do material, em especial ao Anderson A. Araújo (Sapotaceae), Fernando B. Matos (Cyatheaceae), Jomar G. Jardim (Rubiaceae), Marcos Sobral (Cardiopteridaceae e Myrtaceae), Haroldo C. Lima e Marli Morim de Lima (Fabaceae), Pedro Fiaschi (Araliaceae e grupos previamente não identificados em famílias), Pedro Moraes (Lauraceae), Renato Goldenberg (Melastomataceae) e Ricardo O. Perdiz (Meliaceae e Sapindaceae).

\section{Referências bibliográficas}

Amorim, A.M.; Jardim, J.G.; Clifton, B.C.; Fiaschi, P.; Thomas, W.W. \& Carvalho, A.M.V. 2005. The vascular plants of a forest fragment in southern Bahia, Brazil. Sida, Contributions to Botany 21(3): 1726-1752.

Amorim, A.M.; Thomas, W.W.; Carvalho, A.M.V. \& Jardim, J.G. 2008. Floristics of the Una Biological Reserve, Bahia, Brazil. Memoirs of the New York Botanical Garden 100: 67-146.

Amorim, A.M.; Jardim, J.G.; Lopes, M.M.M.; Fiaschi, P.; Borges, R.A.X.; Perdiz, R.O. \& Thomas, W.W. 2009. Angiospermas em remanescentes de floresta Montana no sul da Bahia, Brasil. Biota Neotropica 9(3): 313-348.

Amorim, A.M. \& Leme, E.M.C. 2009. Two New Species of Quesnelia (Bromeliaceae: Bromelioideae) from the Atlantic Rain Forest of Bahia, Brazil. Brittonia 61: 14-21.

APG III - Angiosperm Phylogeny Group. 2009. An update of the Angiosperm Phylogeny Group classification for the orders and families of flowering plants. Botanical Journal of the Linnean Society 161: 105-121.

Cassano, C.R.; Schroth, G.; Faria, D.; Delabie, J.H.C. \& Bede, L. 2008. Landscape and farm scale management to enhance biodiversity conservation in the cocoa producing region of southern Bahia, Brazil. Biodiversity and Conservation 18: 577-603.

Clark, D.B. 1996. Abolishing virginity. Journal of Tropical Ecology 12: 435-439.

Dunning, J.B.; Danielson, B.J. \& Pulliam, H.R. 1992. Ecological processes that affect populations in complex landscapes. Oikos 65(1): 169-175.

Ferraz, E.M.N. \& Rodal, M.J.N. 2006. Caracterização fisionômica-estrutural de um remanescente de floresta ombrófila Montana de Pernambuco, Brasil. Acta Botanica Brasilica 20(4): 911-926. 
Gentry, A.H. 1982. Neotropical Floristic diversity. Annals of the Missouri Botanical Garden 69: 557-593.

Gentry, A.H. 1996. A field guide to the woody plants of Northwest South America. Washington, D.C. Conservation International.

Gotelli, N.J. \& Colwell, R.K. 2001. Quantifying biodiversity: procedures and pitfalls in the measurement and comparison of species richness. Ecology Letters 4: 379-391.

Gotelli, N.J.; Anderson, M.J.; Arita, H.T.; Chao, A.; Colwell, R.K.; Connolly, S.R.; Currie, D.J.; Dunn, R.R.; Graves, G.R.; Green, J.L.; Grytnes, J.A.; Jiang, Y.-H.; Jetz, W.; Lyons, S.K.; McCain, C.M.; Magurran, A.E.; Rahbek, C.; Rangel, T.F.L.V.B.; Soberón, J.; Webb, C.O. \& Willig, M.R. 2009. Patterns and causes of species richness: A general simulation model for macroecology. Ecology Letters 12: 873-886.

Guilherme, F.A.G.; Morellato, L.P.C. \& Assis, M.A. 2004. Horizontal and vertical tree community structure in a lowland Atlantic Rain Forest, Southeastern Brazil. Revista Brasileira de Botânica 27: 725-737.

Hammer, Ø.; Harper, D.A.T. \& Ryan, P.D. 2001. PAST: Paleontological Statistics software package for education and data analysis. Paleontologia Electronica 4(1): 1-9

Kindt, R; Van Damme, P. \& Simons, A.J. 2006. Tree diversity in western Kenya: using profiles to characterize richness and evenness. Biodiversity and Conservation 15: 1253-1270

Kiyoshi, O., Shigeyuki, S. \& Hiroko, F. 1996. Causual analysis of the invasion of broadleaved forest by bamboo in Japan. Journal of Vegetation Science 7: 723-728.

Köppen, W. 1936. Das geographische system der klimatologie. Berlim, Borntrager.

Labiak, P.H. \& Prado, J. 2007. New records of pteridophytes from Bolivia and Brazil. American Fern Journal 97: 113-123.

Lewis, C.E.; Swindel, B.F. \& Tanner, G.W. 1988. Species Diversity and Diversity Profiles: Concept, Measurement, and Application to Timber and Range Management. Journal of Range Management 41(6): 466-469.

Lieberman, D.; Lieberman, M.; Peralta, R. \& Hartshorn, G.S. 1996. Tropical forest and composition on large-scale altitudinal gradient in Costa Rica. Journal of Tropical Ecology 84: 137-152.

Lima, H.C.; Lima, M.P.M.; Vaz, A.M.S.F \& Pessoa, S.V.A. 1997. Trepadeiras da Reserva Ecológica de Macaé de Cima. Pp. 75-88. In: Lima, H.C. \& Guedes-Bruni, R.R. (Eds.). Serra de Macaé de Cima: Diversidade Florística e Conservação em Mata Atlântica. Jardim Botânico do Rio de Janeiro.

Liu, C., Whittaker, R.J., Ma, K. \& Malcolm, J.R. 2007. Unifying and distinguishing diversity ordering methods for comparing communities. Population Ecology 49(2): 89-100.

Magurran, A.E. 1988. Ecological Diversity and its Measurement. Princeton, Princeton University Press.

Magurran, A.E. 2004. Measuring Biological Diversity. Oxford, Blackwell.

Marangon, L.C; Soares, J.J; Feliciano, A.L.P. \& Silva, Brandão, C.F.L.S. 2008. Regeneração natural em um fragmento de floresta estacional semidecidual em Viçosa, Minas Gerais. Revista Árvore 32(1): 183-191.

Martinelli, G. 2007. Mountain biodiversity in Brazil. Revista Brasileira de Botânica 30: 587-597.

Martini, A.M.Z.; Fiaschi, P.; Amorim, A.M. \& Paixão, J.L. 2007. A Hotpoint within a hot-spot: a high diversity site in Brazil's Atlantic Forest. Biodiversity and Conservation 16: 3111-3128.

Matos, F.B. 2009. Samambaias e Licófitas da RPPN Serra Bonita, município de Camacan, sul da Bahia, Brasil. Dissertação de Mestrado, Botânica. Curitiba. Universidade Federal do Paraná.

Matos, F.B.; Labiak, P.H. \& Sylvestre, L.S. 2009. A New Brazilian Species of the Genus Asplenium L. (Aspleniaceae). American Fern Journal 99: 101-105.

Matos, F.B.; Amorim, A.M. \& Labiak, P.H. 2010a. The Ferns and Lycophytes of a Montane Tropical Forest in Southern Bahia, Brazil. Journal of the Botanical Research Institute of Texas 4: 333-346.
Matos, F.B.; Smith, A.R. \& Labiak, P.H. 2010b. A new species of Thelypteris (Thelypteridaceae) from southern Bahia, Brazil. Brittonia 62: 149-152.

Meireles, L.D.; Shepherd, G.J. \& Kinoshita, L.S. 2008. Variações na composição florística e na estrutura fitossociológica de uma floresta ombrófila densa Alto-Montana na Serra da Mantiqueira, Monte Verde, MG. Revista Brasileira de Botânica 31(4): 559-574.

Melo, A.S. 2008. O que ganhamos 'confundindo' riqueza de espécies e equabilidade em um índice de diversidade? Biota Neotropica 8: 21-27.

Mittermeier, R.A., Gil, P.R., Hoffmann, M., Pilgrim, J., Brooks, J., Mittermeier, C.G., Lamourux, J. \& Fonseca, G.A.B. 2004. Hotspots revisited: earth's biologically richest and most endangered terrestrial ecoregions. Washington, Ceme.

Morellato, L.P.C. \& Haddad, C.F.B. 2000. Introduction: The Brazilian Atlantic Forest. Biotropica 32(4b): 786-792.

Moreno, M.R.; Nascimento, M.T. \& Kurtz, B.C. 2003. Estrutura e composição florística do estrato arbóreo em duas zonas altitudinais na mata atlântica de encosta da região do Imbé, RJ. Acta Botanica Brasilica 17(3): 371-386.

Mori, S.A.; Boom, B.M.; Carvalho, A.M. \& Santos, T.S. 1983. Ecological Importance of Myrtaceae in an Eastern Brazilian Wet Forest. Biotropica 15(1): 68-70.

Mori, S.A.; Silva, L.A.M.; Lisboa, G. \& Coradin, L. 1989. Manual de manejo de herbário fanerogâmico. Ilhéus, Centro de Pesquisas do Cacau.

Mota, A.C.; Oliveira, R.P. \& Filgueiras, T.S. 2009. Poaceae de uma área de floresta Montana no sul da Bahia, Brasil: Bambusoideae e Pharoideae. Rodriguésia 60(4): 747-770.

Mortara, S.R. 2011. Estrutura de comunidades de samambaias em uma floresta montana, Bahia, Brasil: diversidade e redes de interação. Dissertação de Mestrado, Ecologia. Universidade Estadual de Santa Cruz, Ilhéus.

Mueller-Dumbois, D. \& Ellenberg, H. 1974 Aims and methods of vegetation ecology. John Wiley \& Sons, New York.

Nabe-Nielsen, J. 2001. Diversity and distribution of lianas in a neotropical rain forest, Yasunõ Â National Park, Ecuador. Journal of Tropical Ecology 17:1-19.

Oliveira-Filho, A.T. \& Fontes, M.A.L. 2000. Patterns of Floristic Differentiation among Atlantic Forests in Southeastern Brazil and the Influence of Climate. Biotropica 32(4b): 793-810.

Oliveira-Filho, A.T.; Jarenkow, J.A. \& Rodal, M.J.N. 2006. Floristic relationships of seasonally dry forests of eastern South America based on tree species distribution patterns. Pp. 59-192. In: Pennington, R.T.; Lewis, G.P. \& Ratter, J.A. (Eds.). Neotropical savannas and seasonally dry forests: plant diversity, biogeography and conservation. Oxford, Francis CRC Press.

Peet, R.K. 1974. The measurement of species diversity. Annual Review of Ecology and Systematics 5: 285-307.

Pendry, C.A. \& Proctor, J. 1996. The causes of altitudinal zonation of rain forests on Bukit Belalong, Brunei. Journal of Ecology 84: 407-418.

Pereira-Silva, E.F.L.; Santos, J.E.; Kageyama, P.Y. \& Hardt, E. 2004. Florística e fitossociologia dos estratos arbustivo e arbóreo de um remanescente de cerradão em uma Unidade de Conservação do estado de São Paulo. Revista Brasileira de Botânica 27(3): 533-544.

Sambuichi, R.H.R. 2002. Fitossociologia e diversidade de espécies arbóreas em cabruca (Mata Atlântica raleada sobre plantação de cacau) na região sul da Bahia, Brasil. Acta Botanica Brasilica 16(1): 89-101.

Shepherd, G.J. 1995. FITOPAC 1. Manual do usuário. Departamento de Botânica. UNICAMP, Campinas.

Silveira, M. 2001. A floresta aberta com bambu no sudoeste da Amazônia: padrões e processos em múltiplas escalas. Tese de Doutorado, Ecologia. Brasília, Universidade de Brasília. 
Tabarelli, M. \& Mantovani, W. 1999. A riqueza da Floresta Atlântica de encosta no Estado de São Paulo (Brasil). Revista Brasileira de Botânica 22: 217-223.

Thomas, W.W.; Carvalho, A.M.V.; Amorim, A.M.; Garrison, J. \& Albeláez, A.L. 1998. Plant endemism in two forests in southern Bahia, Brazil. Biodiversity and Conservation 7: 311-322.

Thomas, W.W.; Carvalho, A.M.V.; Amorim, A.M.; Hanks, J.G. \& Santos, T.S. 2008. Diversity of woody plants in the Atlantic coastal forest of southern Bahia, Brazil. Memoirs of the New York Botanical Garden 100: 21-66.

Thomas, W.W.; Jardim, J.G.; Fiaschi, P.; Mariano-Neto, E. \& Amorim, A.M. 2009. Composição florística e estrutura do componente arbóreo de uma área transicional de Floresta Atlântica no sul da Bahia, Brasil. Revista Brasileira de Botânica 32(1): 65-78.

Tóthmérész, B. 1995. Comparison of Different Methods for Diversity Ordering. Journal of Vegetation Science 6(2): 283-290.

Veblen, T.T. 1982. Growth pattern of Chusquea bamboos in the understory of Chilean Notophagus forests and their influences in forest dynamics. Bulletin of the Torrey Botanical Club 109: 474-487.

Veloso, H.P.; Rangel Filho, A.L.R. \& Lima, J.C.A. 1991. Classificação da vegetação brasileira, adaptada a um sistema universal. Rio de Janeiro: IBGE, Departamento de Recursos Naturais e Estudos Ambientais.

Young, K.R. 1991. Natural History of an Understory Bamboo (Chusquea sp.) in a Tropical Timberline Forest. Biotropica 3(4): 542-554. 